TRANSACTIONS OF THE

AMERICAN MATHEMATICAL SOCIETY

Volume 354, Number 2, Pages 705-733

S 0002-9947(01)02861-6

Article electronically published on September 21, 2001

\title{
TENTH ORDER MOCK THETA FUNCTIONS IN RAMANUJAN'S LOST NOTEBOOK (IV)
}

\author{
YOUN-SEO CHOI
}

\begin{abstract}
Ramanujan's lost notebook contains many results on mock theta functions. In particular, the lost notebook contains eight identities for tenth order mock theta functions. Previously the author proved the first six of Ramanujan's tenth order mock theta function identities. It is the purpose of this paper to prove the seventh and eighth identities of Ramanujan's tenth order mock theta function identities which are expressed by mock theta functions and a definite integral. L. J. Mordell's transformation formula for the definite integral plays a key role in the proofs of these identities. Also, the properties of modular forms are used for the proofs of theta function identities.
\end{abstract}

\section{INTRODUCTION}

In S. Ramanujan's last letter to G. H. Hardy [BR], Ramanujan described a mock theta function, which is a function $f(q)$ defined by a $q$-series which converges for $|q|<1$ and which satisfies the following two conditions:

(1) For every root of unity $\zeta$, there is a theta function $\theta_{\zeta}(q)$ such that the difference $f(q)-\theta_{\zeta}(q)$ is bounded as $q \rightarrow \zeta$ radially.

(2) There is no single theta function which works for all $\zeta$ : i.e., for every theta function $\theta(q)$ there is some root of unity $\zeta$ for which $f(q)-\theta(q)$ is unbounded as $q \rightarrow \zeta$ radially.

He then provided a long list of 'third order,' 'fifth order,' and 'seventh order' mock theta functions together with identities satisfied by them. Further identities can be found in Ramanujan's lost notebook RA].

In his lost notebook [RA p. 9], Ramanujan also gave a list of eight identities involving the following four Ramanujan's tenth order mock theta functions:

$$
\begin{aligned}
& \phi(q):=\sum_{n=0}^{\infty} \frac{q^{n(n+1) / 2}}{\left(q ; q^{2}\right)_{n+1}}, \psi(q):=\sum_{n=0}^{\infty} \frac{q^{(n+1)(n+2) / 2}}{\left(q ; q^{2}\right)_{n+1}}, \\
& X(q):=\sum_{n=0}^{\infty} \frac{(-1)^{n} q^{n^{2}}}{(-q ; q)_{2 n}}, \text { and } \chi(q):=\sum_{n=0}^{\infty} \frac{(-1)^{n} q^{(n+1)^{2}}}{(-q ; q)_{2 n+1}}, \\
& \text { where }(a ; q)_{n}:=\prod_{m=0}^{n-1}\left(1-a q^{m}\right) .
\end{aligned}
$$

The seventh and eighth identities of Ramanujan's identities each expresses a combination of $\phi(q), \psi(q)$, and a definite integral. The main purpose of this paper

Received by the editors August 11, 2000.

2000 Mathematics Subject Classification. Primary 11B65; Secondary 11F20, 33E05.

Key words and phrases. Ramanujan, definite integral, theta function, mock theta function. 
is to prove Ramanujan's seventh and eighth identities. The first six of Ramanujan's identities were proved by the author [C1], C2, C3]. The seventh and eighth identities are

$$
\begin{aligned}
& \int_{0}^{\infty} \frac{e^{-\pi n x^{2}}}{\cosh \frac{2 \pi x}{\sqrt{5}}+\frac{1+\sqrt{5}}{4}} d x+\frac{1}{\sqrt{n}} e^{\frac{\pi}{5 n}} \psi\left(-e^{-\frac{\pi}{n}}\right) \\
& =\sqrt{\frac{5+\sqrt{5}}{2}} e^{-\frac{\pi n}{5}} \phi\left(-e^{-\pi n}\right)-\frac{\sqrt{5}+1}{2 \sqrt{n}} e^{-\frac{\pi}{5 n}} \phi\left(-e^{-\frac{\pi}{n}}\right)
\end{aligned}
$$

and

$$
\begin{aligned}
& \int_{0}^{\infty} \frac{e^{-\pi n x^{2}}}{\cosh \frac{2 \pi x}{\sqrt{5}}+\frac{1-\sqrt{5}}{4}} d x+\frac{1}{\sqrt{n}} e^{\frac{\pi}{5 n}} \psi\left(-e^{-\frac{\pi}{n}}\right) \\
& =-\sqrt{\frac{5-\sqrt{5}}{2}} e^{\frac{\pi n}{5}} \psi\left(-e^{-\pi n}\right)+\frac{\sqrt{5}-1}{2 \sqrt{n}} e^{-\frac{\pi}{5 n}} \phi\left(-e^{-\frac{\pi}{n}}\right) .
\end{aligned}
$$

G. N. Watson WG] also provided several third order mock theta function identities which include the definite integral. Those identities are derived from the generalized Lambert series for the third order mock theta functions by using Cauchy's theorem.

We first need to define a few notations. Throughout the paper, summation indices run through all integers, or through all integers satisfying the conditions listed under the summation sign.

Notation. For a complex number $q$ with $|q|<1$ and $|b c|<1$, set

$$
(a ; q)_{\infty}:=\prod_{m=0}^{\infty}\left(1-a q^{m}\right)
$$

and

$$
f(b, c):=\sum_{j} b^{j(j+1) / 2} c^{j(j-1) / 2} .
$$

We can easily verify the following identity:

$$
\left(a^{2} ; q\right)_{\infty}=\left(a^{2} ; q^{2}\right)_{\infty}\left(a^{2} q ; q^{2}\right)_{\infty}=(-a ; q)_{\infty}(a ; q)_{\infty}\left(a^{2} q ; q^{2}\right)_{\infty} .
$$

Note that

$$
\sum_{j} b^{j(j+1) / 2} c^{j(j-1) / 2}=(-b ; b c)_{\infty}(-c ; b c)_{\infty}(b c ; b c)_{\infty}
$$

is called the Jacobi triple product identity [B1 p. 35], and

$$
f\left(-q,-q^{2}\right)=(q ; q)_{\infty}
$$

is called Euler's pentagonal number theorem [B1, p. 36].

In [C1], the author derived the following results:

$$
\psi(q) f\left(-z,-z^{-1} q^{10}\right)=L_{1}(-q, z)+a_{1}(q) f\left(-z,-z^{-1} q^{10}\right)+2 q A\left(z q^{-2}, q, q^{5}\right)
$$

and

$$
\phi(q) f\left(-z,-z^{-1} q^{10}\right)=L_{2}(-q, z)+a_{2}(q) f\left(-z,-z^{-1} q^{10}\right)+2 q A\left(z q^{-4}, q^{2}, q^{5}\right),
$$


where

$$
\begin{aligned}
L_{1}(-q, z) & =2 \sum_{n} \frac{(-1)^{n} q^{5 n^{2}+5 n} z^{n+1}}{1-q^{10 n+3} z}, \\
L_{2}(-q, z) & =2 \sum_{n} \frac{(-1)^{n} q^{5 n^{2}+5 n-1} z^{n+1}}{1-q^{10 n+1} z}, \\
A(z, x, q) & =\frac{(q ; q)_{\infty}^{2}\left(q^{2} ; q^{2}\right)_{\infty}^{2} f\left(-z x,-z^{-1} x^{-1} q\right)}{f(-q,-q) f\left(-x,-x^{-1} q\right) f\left(-z q,-z^{-1} q\right)}, \\
a_{1}(q) & =-\frac{q\left(q^{5} ; q^{5}\right)_{\infty}\left(q^{10} ; q^{10}\right)_{\infty} f\left(-q^{2},-q^{8}\right)}{f\left(-q,-q^{4}\right) f\left(-q^{4},-q^{6}\right)},
\end{aligned}
$$

and

$$
a_{2}(q)=\frac{\left(q^{5} ; q^{5}\right)_{\infty}\left(q^{10} ; q^{10}\right)_{\infty} f\left(-q^{4},-q^{6}\right)}{f\left(-q^{2},-q^{3}\right) f\left(-q^{2},-q^{8}\right)} .
$$

These two identities (1.7) and (1.8) show that two tenth order mock theta functions can be expressed in terms of generalized Lambert series and theta functions. D. Hickerson [H1], H2 derived similar results for fifth and seventh order mock theta functions, and G. E. Andrews and Hickerson $\mathrm{AH}$ derived similar results for sixth order mock theta functions. The author C2 also derived similar results for the other tenth order mock theta funcions $X(q)$ and $\chi(q)$. We will prove (1.1) and (1.2) with (1.7), (1.8), and the transformation formula for the definite integral [ML]

which is provided by L. J. Mordell. In [ML, p. 333], Mordell provided the following formula related to the definite integral:

$$
\int_{-\infty}^{\infty} \frac{e^{\pi i \omega t^{2}-2 \pi x t}}{e^{2 \pi t}-e^{2 \pi i \theta}} d t=e^{-\pi i\left(\theta^{2} \omega+2 \theta x+2 \theta\right)} \frac{F[(x+\theta \omega) / \omega,-1 / \omega]+i \omega F(x+\theta \omega, \omega)}{\omega \theta_{11}(x+\theta \omega, \omega)},
$$

where

$$
\begin{aligned}
& q=e^{\pi i \omega} \text { with } I(\omega)>0, \\
& i F(x, \omega)=\sum_{m} \frac{(-1)^{m} q^{m^{2}+m+1 / 4} e^{(2 m+1) \pi i x}}{1+q^{2 m+1}},
\end{aligned}
$$

and

$$
i \theta_{11}(x, \omega)=\sum_{m}(-1)^{m} q^{m^{2}+m+1 / 4} e^{(2 m+1) \pi i x} .
$$

In Section 2, we prove nine theta function identities. Six of them are proved by using properties of modular forms. In Section 3, using (1.14), we derive an identity which shows that the definite integral in (1.1) can be expressed by $F(x, \omega)$ and $\theta_{11}(x, \omega)$. Then, applying the generalized Lambert series (1.9) and (1.10), and the two identities (1.7) and (1.8) to the identity, we find that the definite integral in (1.1) is represented by $\phi(q), \psi(q)$, and theta functions. Simplifying the sum of theta functions by using the identities in Section 2, we complete the proof of (1.1). In Section 4, we will also prove Ramanujan's eighth identity by methods similar to those in Section 3. 
The author would like to thank Dr. Andrews and Dr. Berndt. I couldn't have finished the proofs of all eight Ramanujan's identities in page nine of his lost notebook without their help.

\section{Proofs of theta function identities}

In this section, we will prove nine theta function identities. To prove first three identities, we need the next three theorems.

Theorem 2.1. For $0<|q|<1, x \neq 0$, and $y \neq 0$,

$$
\begin{gathered}
f\left(-x,-x^{-1} q\right) f\left(-y,-y^{-1} q\right)=f\left(x y,(x y)^{-1} q^{2}\right) f\left(x^{-1} y q, x y^{-1} q\right) \\
-x f\left(x y q,(x y)^{-1} q\right) f\left(x^{-1} y, x y^{-1} q^{2}\right) .
\end{gathered}
$$

Proof. See Theorem 1.1 in [H1, p. 643].

Theorem 2.2. If $0<|q|<1$ and $a, b, c$, and $d$ are nonzero complex numbers, then

$$
\begin{aligned}
0= & a f\left(-a b,-(a b)^{-1} q\right) f\left(-\frac{b}{a},-\frac{a}{b} q\right) f\left(-c d,-(c d)^{-1} q\right) f\left(-\frac{c}{d},-\frac{d}{c} q\right) \\
& +b f\left(-b c,-(b c)^{-1} q\right) f\left(-\frac{c}{b},-\frac{b}{c} q\right) f\left(-a d,-(a d)^{-1} q\right) f\left(-\frac{a}{d},-\frac{d}{a} q\right) \\
& +c f\left(-a c,-(a c)^{-1} q\right) f\left(-\frac{a}{c},-\frac{c}{a} q\right) f\left(-b d,-(b d)^{-1} q\right) f\left(-\frac{b}{d},-\frac{d}{b} q\right) .
\end{aligned}
$$

Proof. See Theorem 3.5 in [C1, p. 545].

Theorem 2.3. If $a b=c d$, then

$$
f(a, b) f(c, d)+f(-a,-b) f(-c,-d)=2 f(a c, b d) f(a d, b c)
$$

and

$$
f(a, b) f(c, d)-f(-a,-b) f(-c,-d)=2 a f\left(\frac{b}{c}, \frac{c}{b} a b c d\right) f\left(\frac{b}{d}, \frac{d}{b} a b c d\right) .
$$

Proof. See Entry 29 in [B1, p. 45].

Theorem 2.4. If $|q|<1$, then

$$
\begin{aligned}
& \frac{\left(-q^{5} ;-q^{5}\right)_{\infty}\left(q^{10} ; q^{10}\right)_{\infty} f\left(-q^{4},-q^{6}\right)}{f\left(-q^{2}, q^{3}\right) f\left(-q^{2},-q^{8}\right)}-2 q \frac{\left(-q^{5} ;-q^{5}\right)_{\infty}^{2}\left(q^{10} ; q^{10}\right)_{\infty}^{2}}{f\left(q^{5}, q^{5}\right)^{2} f\left(-q^{4},-q^{6}\right)} \\
& =\frac{f\left(-q,-q^{9}\right) f\left(q^{3}, q^{7}\right) f\left(q^{4}, q^{6}\right)}{f\left(-q^{4},-q^{6}\right) f\left(q, q^{9}\right)}
\end{aligned}
$$

Proof. Replacing $q, x$, and $y$ by $q^{5},-q$, and $q^{2}$, respectively, in Theorem 2.1 , we find that

$$
f\left(q, q^{4}\right) f\left(-q^{2},-q^{3}\right)=f\left(-q^{3},-q^{7}\right) f\left(-q^{4},-q^{6}\right)+q f\left(-q,-q^{9}\right) f\left(-q^{2},-q^{8}\right) .
$$

Replacing $q, a, b, c$, and $d$ by $q^{10}, q^{3}, q^{2}, q^{5}$, and $q$, respectively, in Theorem 2.2, we find that

$$
\begin{aligned}
& f\left(-q^{2},-q^{8}\right) f\left(-q^{3},-q^{7}\right)^{2} f\left(-q^{4},-q^{6}\right) \\
& =f\left(-q,-q^{9}\right) f\left(-q^{4},-q^{6}\right)^{2} f\left(-q^{5},-q^{5}\right)+q f\left(-q,-q^{9}\right) f\left(-q^{2},-q^{8}\right)^{2} f\left(-q^{3},-q^{7}\right) .
\end{aligned}
$$


Multiplying both sides of (2.1) by $f\left(-q^{2},-q^{8}\right) f\left(-q^{3},-q^{7}\right)$, and using (2.2), we find that

$$
\begin{aligned}
& f\left(-q^{2},-q^{8}\right) f\left(-q^{3},-q^{7}\right) f\left(q, q^{4}\right) f\left(-q^{2},-q^{3}\right) \\
& =f\left(-q,-q^{9}\right) f\left(-q^{4},-q^{6}\right)^{2} f\left(-q^{5},-q^{5}\right)+2 q f\left(-q,-q^{9}\right) f\left(-q^{2},-q^{8}\right)^{2} f\left(-q^{3},-q^{7}\right) .
\end{aligned}
$$

Now, dividing both sides of (2.3) by

$$
f\left(-q,-q^{9}\right) f\left(-q^{2},-q^{8}\right)^{2} f\left(-q^{3},-q^{7}\right) f\left(-q^{4},-q^{6}\right) f\left(-q^{5},-q^{5}\right) /\left(q^{10} ; q^{10}\right)_{\infty}^{3},
$$

using (1.4), and replacing $q$ by $-q$, we complete the proof of theorem.

Theorem 2.5. If $|q|<1$, then

$$
\begin{aligned}
& -\frac{\left(-q^{5} ;-q^{5}\right)_{\infty}\left(q^{10} ; q^{10}\right)_{\infty} f\left(-q^{2},-q^{8}\right)}{f\left(q,-q^{4}\right) f\left(-q^{4},-q^{6}\right)}+2 \frac{\left(-q^{5} ;-q^{5}\right)_{\infty}^{2}\left(q^{10} ; q^{10}\right)_{\infty}^{2}}{f\left(q^{5}, q^{5}\right)^{2} f\left(-q^{2},-q^{8}\right)} \\
& =\frac{f\left(-q^{3},-q^{7}\right) f\left(q, q^{9}\right) f\left(q^{2}, q^{8}\right)}{f\left(-q^{2},-q^{8}\right) f\left(q^{3}, q^{7}\right)} .
\end{aligned}
$$

Proof. Replacing $q, x$, and $y$ by $q^{5}, q$, and $-q^{3}$, respectively, in Theorem 2.1, we find that

$$
f\left(-q,-q^{4}\right) f\left(q^{2}, q^{3}\right)=f\left(-q^{4},-q^{6}\right) f\left(-q^{3},-q^{7}\right)-q f\left(-q,-q^{9}\right) f\left(-q^{2},-q^{8}\right) .
$$

Replacing $q, a, b, c$, and $d$ by $q^{10}, q^{4}, q, q^{5}$, and $q^{3}$, respectively, in Theorem 2.2, and dividing both sides of Theorem 2.2 by $q$, we find that

$$
\begin{aligned}
& q f\left(-q,-q^{9}\right)^{2} f\left(-q^{2},-q^{8}\right) f\left(-q^{4},-q^{6}\right) \\
& =f\left(-q^{2},-q^{8}\right)^{2} f\left(-q^{3},-q^{7}\right) f\left(-q^{5},-q^{5}\right)-f\left(-q,-q^{9}\right) f\left(-q^{3},-q^{7}\right) f\left(-q^{4},-q^{6}\right)^{2} .
\end{aligned}
$$

Multiplying both sides of (2.4) by $f\left(-q,-q^{9}\right) f\left(-q^{4},-q^{6}\right)$, and using (2.5), we find that

$$
\begin{aligned}
& f\left(-q,-q^{9}\right) f\left(-q^{4},-q^{6}\right) f\left(-q,-q^{4}\right) f\left(q^{2}, q^{3}\right) \\
& =2 f\left(-q,-q^{9}\right) f\left(-q^{3},-q^{7}\right) f\left(-q^{4},-q^{6}\right)^{2}-f\left(-q^{2},-q^{8}\right)^{2} f\left(-q^{3},-q^{7}\right) f\left(-q^{5},-q^{5}\right) .
\end{aligned}
$$

Now, dividing both sides of (2.6) by

$$
f\left(-q,-q^{9}\right) f\left(-q^{2},-q^{8}\right) f\left(-q^{3},-q^{7}\right) f\left(-q^{4},-q^{6}\right)^{2} f\left(-q^{5},-q^{5}\right) /\left(q^{10} ; q^{10}\right)_{\infty}^{3},
$$

using (1.4), and replacing $q$ by $-q$, we complete the proof of theorem.

Theorem 2.6. If $|q|<1$, then

$$
\begin{aligned}
& f\left(-q^{15},-q^{35}\right) f\left(q^{25}, q^{25}\right)+q^{3} f\left(-q^{5},-q^{45}\right) f\left(q^{25}, q^{25}\right) \\
& +f\left(-q^{25},-q^{25}\right) f\left(q^{15}, q^{35}\right)-q^{4} f\left(-q^{5},-q^{45}\right) f\left(q^{15}, q^{35}\right) \\
& -q^{3} f\left(-q^{25},-q^{25}\right) f\left(q^{5}, q^{45}\right)-q^{4} f\left(-q^{15},-q^{35}\right) f\left(q^{5}, q^{45}\right) \\
& =2 f\left(-q^{4},-q^{16}\right) f\left(-q^{8},-q^{12}\right) .
\end{aligned}
$$

Proof. Let $a b=c d=q^{50}$ in Theorem 2.3. Replacing $a$ and $c$ by $-q^{15}$ and $q^{25}$, respectively, in Theorem 2.3 (i), we find that

$$
f\left(-q^{15},-q^{35}\right) f\left(q^{25}, q^{25}\right)+f\left(-q^{25},-q^{25}\right) f\left(q^{15}, q^{35}\right)=2 f\left(-q^{40},-q^{60}\right)^{2} .
$$


Replacing $a$ and $c$ by $-q^{5}$ and $q^{25}$, respectively, in Theorem 2.3 (ii), we find that

$$
f\left(-q^{5},-q^{45}\right) f\left(q^{25}, q^{25}\right)-f\left(-q^{25},-q^{25}\right) f\left(q^{5}, q^{45}\right)=-2 q^{5} f\left(-q^{20},-q^{80}\right)^{2} .
$$

Replacing $a$ and $c$ by $-q^{5}$ and $q^{15}$, respectively, in Theorem 2.3 (i), we find that

$$
f\left(-q^{5},-q^{45}\right) f\left(q^{15}, q^{35}\right)+f\left(-q^{15},-q^{35}\right) f\left(q^{5}, q^{45}\right)=2 f\left(-q^{20},-q^{80}\right) f\left(-q^{40},-q^{60}\right) .
$$

Thus, by (2.8), (2.9), and (2.10), the left side of (2.7) equals

$$
2 f\left(-q^{40},-q^{60}\right)^{2}-2 q^{8} f\left(-q^{20},-q^{80}\right)^{2}-2 q^{4} f\left(-q^{20},-q^{80}\right) f\left(-q^{40},-q^{60}\right) .
$$

Now, in [B2] p. 191], we find that, for $|x|<1$,

$$
\begin{aligned}
f(x A, x / A) f(x B, x / B) & =f\left(x^{5} A^{2} B, x^{5} A^{-2} B^{-1}\right) f\left(x^{5} A^{-1} B^{2}, x^{5} A B^{-2}\right) \\
& +x B f\left(x^{7} A^{2} B, x^{3} A^{-2} B^{-1}\right) f\left(x^{9} A^{-1} B^{2}, x A B^{-2}\right) \\
& +x B^{-1} f\left(x^{3} A^{2} B, x^{7} A^{-2} B^{-1}\right) f\left(x A^{-1} B^{2}, x^{9} A B^{-2}\right) \\
& +x A f\left(x^{9} A^{2} B, x A^{-2} B^{-1}\right) f\left(x^{3} A^{-1} B^{2}, x^{7} A B^{-2}\right) \\
& +x A^{-1} f\left(x A^{2} B, x^{9} A^{-2} B^{-1}\right) f\left(x^{7} A^{-1} B^{2}, x^{3} A B^{-2}\right) .
\end{aligned}
$$

Multiplying both sides of (2.11) by 2 , and replacing $x, A$, and $B$ by $-q^{10}, q^{6}$, and $q^{-2}$, respectively, in $(2.11)$, we find that

$$
\begin{aligned}
& 2 f\left(-q^{4},-q^{16}\right) f\left(-q^{8},-q^{12}\right) \\
& =2 f\left(-q^{40},-q^{60}\right)^{2}-2 q^{8} f\left(-q^{20},-q^{80}\right)^{2}-2 q^{4} f\left(-q^{20},-q^{80}\right) f\left(-q^{40},-q^{60}\right) .
\end{aligned}
$$

Thus, we have completed the proof of this theorem.

We will prove six eta-function identities by using the properties of modular forms.

Definition of the Dedekind eta-function. Let $\mathbf{H}=\{z: \operatorname{Im} z>0\}$. For $z \in$ $\mathbf{H}, q=e^{2 \pi i z}$ and any positive integer $n$, define

$$
\eta(n z):=\eta_{n}=e^{\frac{\pi i n z}{12}} \prod_{m=1}^{\infty}\left(1-e^{2 \pi i m n z}\right)=q^{\frac{n}{24}}\left(q^{n} ; q^{n}\right)_{\infty}=q^{\frac{n}{24}} f\left(-q^{n},-q^{2 n}\right)
$$

Definition of the generalized Dedekind eta-function. Let $\mathbf{H}=\{z: \operatorname{Im} z$ $>0\}$. For $z \in \mathbf{H}, q=e^{2 \pi i z}$, and any positive integers $n, m$, define

$$
\begin{aligned}
\eta_{n, m}(z):=\eta_{n, m} & =e^{\pi i P_{2}\left(\frac{m}{n}\right) n z} \prod_{\substack{k=1 \\
k \equiv m(\bmod n)}}^{\infty}\left(1-e^{2 \pi i k z}\right) \prod_{\substack{k=1 \\
k \equiv-m(\bmod n)}}^{\infty}\left(1-e^{2 \pi i k z}\right) \\
& =q^{P_{2}\left(\frac{m}{n}\right) \frac{n}{2}} \frac{f\left(-q^{m},-q^{n-m}\right)}{\left(q^{n} ; q^{n}\right)_{\infty}},
\end{aligned}
$$

where $P_{2}(t)=\{t\}^{2}-\{t\}+\frac{1}{6}$ is the second Bernoulli function, and $\{t\}:=t-[t]$ is the fractional part of $t$.

In this paper, we only consider the cases with $m \not \equiv 0(\bmod n)$ for $\eta_{n, m}$.

Definition of the modular group. The modular group is the set of linear fractional transformations $T, T(z)=\frac{a z+b}{c z+d}$, where $a, b, c$, and $d$ are rational integers such that $a d-b c=1$. The modular group is denoted by $\Gamma(1)$. Let $\Gamma_{1}(N)$, where $N$ is a positive integer, be the set of linear factional transformations $U, U(z)=\frac{a z+b}{c z+d}$, 
where $a, b, c$, and $d$ are rational integers such that $a d-b c=1, c \equiv 0(\bmod N)$, and $a \equiv d \equiv 1(\bmod N)$.

Clearly, $\Gamma_{1}(N)$ is a subgroup of $\Gamma(1)$.

Definition of a fundamental region. Let $\Gamma$ be a subgroup of $\Gamma(1)$. A fundamental region for $\Gamma$ is an open subset $R$ of $\mathbf{H}$ such that (a) for any two distinct points $z_{1}, z_{2}$ in $R$, there is no $T \in \Gamma$ such that $T\left(z_{1}\right)=z_{2}$, and (b) for any point $z$ in $\mathbf{H}$, there is some point $z_{3}$ in the closure of $R$ such that $T^{\prime}(z)=z_{3}$ for some $T^{\prime} \in \Gamma$.

Definition of a standard fundamental region. Let $\Gamma$ be a subgroup of $\Gamma(1)$ with cosets $A_{1}, A_{2}, \ldots, A_{\mu}$ in the sense that $\Gamma(1)=\bigcup_{i=1}^{\mu} \Gamma A_{i}$. Then

$$
R=\bigcup_{i=1}^{\mu} A_{i} \quad(\text { a fundamental region for } \Gamma(1))
$$

is called a standard fundamental region for $\Gamma$.

Definition of a cusp. Let $R$ be a fundamental region of $\Gamma$. A parabolic cusp of $\Gamma$ in $R$ is any real point $q$, or $q=\infty$, such that $q \in \bar{R}$, the closure of $R$ in the topology of the Riemann sphere.

Definition of a modular form of weight $r$. Let $r$ be a real number. A function $F(z)$, defined and meromorphic in $\mathbf{H}$, is said to be a modular form of weight $r$ with respect to $\Gamma$, with multiplier system $v$, if

(a) $F(z)$ satisfies $F(M z)=v(M)(c z+d)^{r} F(z)$ for any $z \in \mathbf{H}$ and $M \in \Gamma$,

(b) there exists a standard fundamental region $R$ such that $F(z)$ has at most finitely many poles in $\bar{R} \cap \mathbf{H}$, and

(c) $F(z)$ is meromorphic at $q_{j}$, for each cusp $q_{j}$ in $\bar{R}$.

The multiplier system $v=v(M)$ for the group $\Gamma$ is a complex-valued function of absolute value 1 satisfying the equation

$$
v\left(M_{1} M_{2}\right)\left(c_{3} z+d_{3}\right)^{r}=v\left(M_{1}\right) v\left(M_{2}\right)\left(c_{1} M_{2} z+d_{1}\right)^{r}\left(c_{2} z+d_{2}\right)^{r}
$$

for $M_{1}, M_{2} \in \Gamma$, where $M_{1}(z)=\frac{a_{1} z+b_{1}}{c_{1} z+d_{1}}, M_{2}(z)=\frac{a_{2} z+b_{2}}{c_{2} z+d_{2}}$, and $M_{3}(z)=$ $\left(M_{1} M_{2}\right)(z)=\frac{a_{3} z+b_{3}}{c_{3} z+d_{3}}$.

Let $\{\Gamma, r, v\}$ denote the space of modular forms of weight $r$ and multiplier system $v$ on $\Gamma$, where $\Gamma$ is a subgroup of $\Gamma(1)$ of finite index.

Let $\operatorname{ord}(f ; z)$ denote the invariant order of a modular form $f$ at $z$. Let $\operatorname{Ord}_{\Gamma}(f ; z)$ denote the order of $f$ with respect to $\Gamma$, defined by $\operatorname{Ord}_{\Gamma}(f ; z):=\frac{1}{\ell} \operatorname{ord}(f ; z)$, where $\ell$ is the order of $f$ at $z$ as a fixed point of $\Gamma$.

Theorem 2.7. The Dedekind eta-function $\eta(z)$ is a modular form of weight $1 / 2$ on the full modular group $\Gamma(1)$.

Proof. See Theorem 10 in [KM, p. 43]. 
Theorem 2.8. The multiplier system $v_{\eta}$ of the modular form $\eta(z)$ is given by the following formula: for each $M=\left(\begin{array}{ll}a & b \\ c & d\end{array}\right) \in \Gamma(1)$,

$v_{\eta}(M)= \begin{cases}\left(\frac{d}{|c|}\right) \zeta_{24}^{b d\left(1-c^{2}\right)+c(a+d)-3 c}, & \text { if } c \text { is odd, } \\ \left(\frac{c}{|d|}\right) \zeta_{24}^{a c\left(1-d^{2}\right)+d(b-c)+3(d-1)}, & \text { if } d \text { is odd and either } c \geq 0 \text { or } d \geq 0, \\ -\left(\frac{c}{|d|}\right) \zeta_{24}^{a c\left(1-d^{2}\right)+d(b-c)+3(d-1)}, & \text { if } d \text { is odd, } c<0, d<0,\end{cases}$

where $\zeta_{24}$ is a primitive 24 th root of unity.

Proof. See Theorem 2 in [KM, p. 51].

Theorem 2.9 (The valence formula). If $f \in\{\Gamma, r, \nu\}$ and $f \neq 0$, then

$$
\sum_{z \in R} \operatorname{Ord}_{\Gamma}(f ; z)=\mu r
$$

where $R$ is any fundamental region for $\Gamma$, and $\mu:=\frac{1}{12}[\Gamma(1): \Gamma]$.

Proof. See Theorem 4.1.4 in $[\mathrm{RR}$.

Lemma 2.10. If $m_{1}, m_{2}, \ldots, m_{2 n}$ are positive integers, $n$ is a positive integer, $N$ is a positive even integer, and the least common multiple of $m_{1}, m_{2}, \ldots, m_{2 n}$ divides $N$, then, for $z \in \mathbf{H}, \eta\left(m_{1} z\right) \eta\left(m_{2} z\right) \cdots \eta\left(m_{2 n} z\right) \in\left\{\Gamma_{1}(N), n, v\right\}$, where $A=\left(\begin{array}{ll}a & b \\ c & d\end{array}\right) \in \Gamma_{1}(N), \zeta_{24}$ is a primitive 24th root of unity, and

$$
v(A)=\prod_{i=1}^{2 n}\left(\frac{c / m_{i}}{|d|}\right) \zeta_{24}^{a c\left(1-d^{2}\right) / m_{i}+d\left(m_{i} b-c / m_{i}\right)+3(d-1)}
$$

Proof. See Lemma 2.2.4 in [C3].

Lemma 2.11. For a positive integer $n,\left[\Gamma(1): \Gamma_{1}(n)\right]=n^{2} \prod_{p \mid n}\left(1-\frac{1}{p^{2}}\right)$, where the product is over all primes $p$ dividing $n$.

Proof. See Lemma 2.6.5 in [C2].

Theorem 2.12. For $z \in \mathbf{H}$, let $f(z):=\prod_{\substack{n \mid N \\ 0 \leq m<n}} \eta_{n, m}^{r_{n}, m}(z)$, where $r_{n, m}$ are integers.

$$
\text { If } \sum_{\substack{n \mid N \\ 0 \leq m<n}} n P_{2}\left(\frac{m}{n}\right) r_{n, m} \equiv 0 \quad(\bmod 2) \text { and } \sum_{\substack{n \mid N \\ 0 \leq m<n}} \frac{N}{n} P_{2}(0) r_{n, m} \equiv 0 \quad(\bmod 2) \text {, }
$$

then $f(z) \in\left\{\Gamma_{1}(N), 0, I\right\}$, where for $M=\left(\begin{array}{ll}a & b \\ c & d\end{array}\right) \in \Gamma_{1}(N), I(M)=1$.

Proof. See Theorem 3 in [RS, p. 126]. 
Theorem 2.13. For $z \in \mathbf{H}$,

$$
\begin{aligned}
& \eta_{50}^{18} \eta_{10,2}^{4} \eta_{10,3} \eta_{20,2} \eta_{20,4} \eta_{100,50} \\
& \times\left(-\eta_{50,5} \eta_{50,10}^{2} \eta_{50,15} \eta_{50,25} \eta_{100,40}+\eta_{50,5} \eta_{50,10} \eta_{50,15} \eta_{50,20} \eta_{50,25} \eta_{100,40}\right. \\
&-\eta_{50,15}^{2} \eta_{50,20}^{2} \eta_{50,25} \eta_{100,20}+2 \eta_{50,5} \eta_{50,15} \eta_{50,20}^{2} \eta_{50,25} \eta_{100,40} \\
&\left.-2 \eta_{50,5}^{2} \eta_{50,20} \eta_{50,25} \eta_{100,30} \eta_{100,40}^{2}+\eta_{50,5}^{2} \eta_{50,15} \eta_{50,20} \eta_{100,40}^{2} \eta_{100,50}\right) \\
&= \eta_{10}^{2} \eta_{100}^{16} \eta_{10,1}^{2} \eta_{10,2}^{4} \eta_{20,8}^{2} \eta_{50,10} \eta_{50,15} \eta_{50,20}^{2} \eta_{50,25} \eta_{100,50}^{9} .
\end{aligned}
$$

Proof. For $1 \leq i \leq 7$, let $f_{i}^{1}$ be the product of eta-functions in each of the 7 products in (2.14), and $g_{i}^{1}$ be the product of the generalized eta-functions in each of the 7 products in (2.14). Each $f_{i}^{1}$ is the product of 18 eta-functions, and by Theorem 2.10 and a straightforward calculation, each $f_{i}^{1}$ is a modular form of weight 9 on $\Gamma_{1}(300)$ with the multiplier system $v_{1}$, where for $A=\left(\begin{array}{ll}a & b \\ c & d\end{array}\right) \in \Gamma_{1}(300), v_{1}(A)=$ $\zeta_{24}^{\frac{1}{25} c\left(a-a d^{2}-d\right)+12 b d+3(d-1)}$. By Theorem 2.12 and a straightforward calculation, each $g_{i}^{1}$ is a modular form of weight 0 on $\Gamma_{1}(300)$ with the multiplier system $I$. Therefore, each $f_{i}^{1} g_{i}^{1}$ is a modular form of weight 9 on $\Gamma_{1}(300)$ with multiplier system $v_{1}$. By Theorem 2.11, $\left[\Gamma(1): \Gamma_{1}(300)\right]=57600$. Let $F_{1}$ denote the difference of the left and right sides of (2.14). Applying Theorem 2.9 (the valence formula), for a fundamental region $R$ for $\Gamma_{1}(300)$, we deduce that, for $F_{1}$,

$$
\sum_{z \in R} \operatorname{Ord}_{\Gamma_{1}(300)}\left(F_{1} ; z\right)=\frac{9 \cdot 57600}{12}=43200 \geq \operatorname{ord}\left(F_{1} ; \infty\right),
$$

since both sides of (2.14) are analytic on $R$. Using Mathematica, we calculated the Taylor series of $F_{1}$ about $q=0$ (or about the cusp $z=\infty$ ) and found that $F_{1}=O\left(q^{43201}\right)$. Unless $F_{1}$ is a constant, we have a contradiction to $(2.15)$. We have thus completed the proof of Theorem 2.13.

Theorem 2.14. For $z \in \mathbf{H}$,

$$
\begin{aligned}
\eta_{50}^{18} \eta_{10,1} & \eta_{10,2}^{16} \eta_{20,6} \eta_{20,8} \eta_{100,50} \\
\times & \left(\eta_{50,5} \eta_{50,10} \eta_{50,15} \eta_{50,20} \eta_{50,25} \eta_{100,20}+\eta_{50,5} \eta_{50,15} \eta_{50,20}^{2} \eta_{50,25} \eta_{100,20}\right. \\
& -\eta_{50,5}^{2} \eta_{50,10}^{2} \eta_{50,25} \eta_{100,40}-2 \eta_{50,5} \eta_{50,10}^{2} \eta_{50,15} \eta_{50,25} \eta_{100,20} \\
& \left.-2 \eta_{50,10} \eta_{50,15}^{2} \eta_{50,25} \eta_{100,10} \eta_{100,20}^{2}+\eta_{50,5} \eta_{50,10} \eta_{50,15}^{2} \eta_{100,20}^{2} \eta_{100,50}\right) \\
= & \eta_{10}^{2} \eta_{100}^{16} \eta_{10,2}^{16} \eta_{10,3}^{2} \eta_{20,4} \eta_{50,5} \eta_{50,10}^{2} \eta_{50,20} \eta_{50,25} \eta_{100,50}^{9} .
\end{aligned}
$$

Proof. For $1 \leq i \leq 7$, let $f_{i}^{2}$ be the product of eta-functions in each of the 7 products in (2.16), and $g_{i}^{2}$ be the product of the generalized eta-functions in each of the 7 products in (2.16). Each $f_{i}^{2}$ is the product of 18 eta-functions, and by Theorem 2.10 and a straightforward calculation, each $f_{i}^{2}$ is a modular form of weight 9 on $\Gamma_{1}(300)$ with the multiplier system $v_{2}$, where for $A=\left(\begin{array}{ll}a & b \\ c & d\end{array}\right) \in \Gamma_{1}(300), v_{2}(A)=$ $\zeta_{24}^{\frac{1}{24} c\left(a-a d^{2}-d\right)+12 b d+3(d-1)}$. By Theorem 2.12 and a straightforward calculation, each $g_{i}^{2}$ is a modular form of weight 0 on $\Gamma_{1}(300)$ with the multiplier system $I$. Therefore, each $f_{i}^{2} g_{i}^{2}$ is a modular form of weight 9 on $\Gamma_{1}(300)$ with multiplier system $v_{2}$. Let $F_{2}$ denote the difference of the left and right sides of (2.16). Applying Theorem 
2.9 , for a fundamental region $R$ for $\Gamma_{1}(300)$, we deduce that, for $F_{2}$,

$$
\sum_{z \in R} \operatorname{Ord}_{\Gamma_{1}(300)}\left(F_{2} ; z\right)=\frac{9 \cdot 57600}{12}=43200 \geq \operatorname{ord}\left(F_{2} ; \infty\right),
$$

since both sides of (2.16) are analytic on $R$. Using Mathematica, we calculated the Taylor series of $F_{2}$ about $q=0$ (or about the cusp $z=\infty$ ) and found that $F_{2}=O\left(q^{43201}\right)$. Unless $F_{2}$ is a constant, we have a contradiction to (2.6). We have therefore completed the proof of Theorem 2.14.

Theorem 2.15. For $z \in \mathbf{H}$,

$$
\begin{aligned}
& \eta_{5}^{2} \eta_{100}^{2} \eta_{5,1}^{10} \eta_{6,1}^{3} \\
& \times\left(\eta_{50,5} \eta_{50,10}^{3} \eta_{100,40} \eta_{100,50}+\eta_{50,15} \eta_{50,20}^{3} \eta_{100,20} \eta_{100,50}-3 \eta_{50,10}^{2} \eta_{50,20}^{2} \eta_{100,25}^{2}\right) \\
& =\eta_{20}^{2} \eta_{25}^{2} \eta_{5,1}^{10} \eta_{6,1}^{3} \eta_{20,5}^{2} \eta_{25,5}^{2} \eta_{25,10}^{2} \eta_{50,10}^{2} \eta_{50,20}^{2} .
\end{aligned}
$$

Proof. For $1 \leq i \leq 4$, let $f_{i}^{3}$ be the product of eta-functions in each of the 4 products in (2.18), and $g_{i}^{3}$ be the product of the generalized eta-functions in each of the 4 products in (2.18). Each $f_{i}^{3}$ is the product of 14 eta-functions, and by Theorem 2.10 and a straightforward calculation, each $f_{i}^{3}$ is a modular form of weight 2 on $\Gamma_{1}(300)$ with the multiplier system $v_{3}$, where for $A=\left(\begin{array}{ll}a & b \\ c & d\end{array}\right) \in \Gamma_{1}(300), v_{3}(A)=$

$\zeta_{24}^{\frac{1}{50} c\left(a-a d^{2}-d\right)+18 b d+3(d-1)}$. By Theorem 2.12 and a straightforward calculation, each $g_{i}^{3}$ is a modular form of weight 0 on $\Gamma_{1}(300)$ with the multiplier system $I$. Therefore, each $f_{i}^{3} g_{i}^{3}$ is a modular form of weight 2 on $\Gamma_{1}(300)$ with multiplier system $v_{3}$. Let $F_{3}$ denote the difference of the left and right sides of (2.18). Applying Theorem 2.9 , for a fundamental region $R$ for $\Gamma_{1}(300)$, we deduce that, for $F_{3}$,

$$
\sum_{z \in R} \operatorname{Ord}_{\Gamma_{1}(300)}\left(F_{3} ; z\right)=\frac{2 \cdot 57600}{12}=9600 \geq \operatorname{ord}\left(F_{3} ; \infty\right),
$$

since both sides of (2.18) are analytic on $R$. Using Mathematica, we calculated the Taylor series of $F_{3}$ about $q=0$ (or about the cusp $z=\infty$ ) and found that $F_{3}=O\left(q^{9601}\right)$. Unless $F_{3}$ is a constant, we have a contradiction to (2.19). We have thus completed the proof of Theorem 2.15.

Theorem 2.16. For $z \in \mathbf{H}$,

$$
\begin{aligned}
& \eta_{4}^{2} \eta_{50}^{2} \eta_{10,1}^{2} \eta_{10,2}^{3} \eta_{10,3} \eta_{20,2} \\
& \times\left(\eta_{50,5} \eta_{50,15} \eta_{50,25} \eta_{100,50}+\eta_{50,5} \eta_{50,15} \eta_{50,25} \eta_{100,30}-\eta_{50,5} \eta_{50,15} \eta_{50,25} \eta_{100,10}\right. \\
& \left.-\eta_{50,5}^{2} \eta_{50,15} \eta_{100,50}-\eta_{50,5} \eta_{50,25}^{2} \eta_{100,30}+2 \eta_{50,5}^{2} \eta_{50,25} \eta_{100,30}-\eta_{50,15}^{2} \eta_{50,25} \eta_{100,10}\right) \\
& =\eta_{10}^{2} \eta_{20}^{2} \eta_{10,1}^{3} \eta_{10,2}^{3} \eta_{20,4}^{3} \eta_{20,8}^{3} \eta_{50,5} \eta_{50,15} \eta_{50,25} .
\end{aligned}
$$

Proof. For $1 \leq i \leq 8$, let $f_{i}^{4}$ be the product of eta-functions in each of the 8 products in (2.20), and $g_{i}^{4}$ be the product of the generalized eta-functions in each of the 8 products in (2.20). Each $f_{i}^{4}$ is the product of 4 eta-functions, and by Theorem 2.10 and a straightforward calculation, each $f_{i}^{4}$ is a modular form of weight 2 on $\Gamma_{1}(300)$ with the multiplier system $v_{4}$, where for $A=\left(\begin{array}{ll}a & b \\ c & d\end{array}\right) \in \Gamma_{1}(300), v_{4}(A)=$ 
$\zeta_{24}^{\frac{3}{50} c\left(a-a d^{2}-d\right)+12 b d+3(d-1)}$. By Theorem 2.12 and a straightforward calculation, each $g_{i}^{4}$ is a modular form of weight 0 on $\Gamma_{1}(300)$ with the multiplier system $I$. Therefore, each $f_{i}^{4} g_{i}^{4}$ is a modular form of weight 2 on $\Gamma_{1}(300)$ with multiplier system $v_{4}$. Let $F_{4}$ denote the difference of the left and right sides of (2.20). Applying Theorem 2.9 , for a fundamental region $R$ for $\Gamma_{1}(300)$, we deduce that, for $F_{4}$,

$$
\sum_{z \in R} \operatorname{Ord}_{\Gamma_{1}(300)}\left(F_{4} ; z\right)=\frac{2 \cdot 57600}{12}=9600 \geq \operatorname{ord}\left(F_{4} ; \infty\right),
$$

since both sides of (2.20) are analytic on $R$. Using Mathematica, we calculated the Taylor series of $F_{4}$ about $q=0$ (or about the cusp $z=\infty$ ) and found that $F_{4}=O\left(q^{9601}\right)$. Unless $F_{4}$ is a constant, we have a contradiction to $(2.21)$. We have thus completed the proof of Theorem 2.16.

Theorem 2.17. For $z \in \mathbf{H}$,

$$
\begin{aligned}
\eta_{10}^{8} & \eta_{20}^{3} \eta_{50} \eta_{10,1} \eta_{10,4} \eta_{20,4} \eta_{20,8}^{2} \eta_{50,5} \eta_{50,15} \eta_{50,25} \eta_{200,50} \eta_{200,100} \\
& \times\left(\eta_{10,1} \eta_{20,10} \eta_{50,10} \eta_{100,40}-4 \eta_{10,3} \eta_{20,2} \eta_{50,10} \eta_{100,40}\right. \\
& \left.+\eta_{10,1} \eta_{20,10} \eta_{50,20} \eta_{100,20}+6 \eta_{10,3} \eta_{20,2} \eta_{50,20} \eta_{100,20}\right) \\
- & 4 \eta_{10,1} \eta_{10,4} \eta_{20,4} \eta_{20,8}^{2} \eta_{40,10} \eta_{50,5} \eta_{50,10} \eta_{50,15} \eta_{50,20} \eta_{50,25} \eta_{200,100} \\
& \times\left(\eta_{10}^{7} \eta_{20}^{2} \eta_{50}^{2} \eta_{100} \eta_{10,1} \eta_{50,10}^{2} \eta_{50,20}^{2}+\eta_{10} \eta_{20}^{10} \eta_{100} \eta_{10,3} \eta_{20,2} \eta_{20,10}^{3}\right) \\
= & \eta_{10}^{8} \eta_{20}^{3} \eta_{50} \eta_{50,5} \eta_{50,10} \eta_{50,15} \eta_{50,20} \eta_{100,50} \eta_{200,50} \eta_{200,100} \\
\times & \left(\eta_{10,1}^{3} \eta_{20,6} \eta_{20,8}^{2} \eta_{20,10}-4 \eta_{10,1} \eta_{10,3} \eta_{10,4} \eta_{20,2} \eta_{20,5}^{2} \eta_{20,8}-\eta_{10,3}^{3} \eta_{20,2} \eta_{20,4}^{2} \eta_{20,10}\right) \\
+ & \eta_{10}^{8} \eta_{20}^{3} \eta_{50} \eta_{50,5} \eta_{50,10} \eta_{50,20} \eta_{50,25} \eta_{100,30} \eta_{200,50} \eta_{200,100} \\
\times & \left(2 \eta_{10,1}^{3} \eta_{20,6} \eta_{20,8}^{2} \eta_{20,10}+2 \eta_{10,1} \eta_{10,3} \eta_{10,4} \eta_{20,2} \eta_{20,5}^{2} \eta_{20,8}+3 \eta_{10,3}^{3} \eta_{20,2} \eta_{20,4}^{2} \eta_{20,10}\right) \\
- & \eta_{10}^{8} \eta_{20}^{3} \eta_{50} \eta_{50,10} \eta_{50,15} \eta_{50,20} \eta_{50,25} \eta_{100,10} \eta_{200,50} \eta_{200,100} \\
\times & \left(3 \eta_{10,1}^{3} \eta_{20,6} \eta_{20,8}^{2} \eta_{20,10}-2 \eta_{10,1} \eta_{10,3} \eta_{10,4} \eta_{20,2} \eta_{20,5}^{2} \eta_{20,8}+2 \eta_{10,3}^{3} \eta_{20,2} \eta_{20,4}^{2} \eta_{20,10}\right)
\end{aligned}
$$

Proof. For $1 \leq i \leq 15$, let $f_{i}^{5}$ be the product of eta-functions in each of the 15 products in $(2.22)$, and $g_{i}^{5}$ be the product of the generalized eta-functions in each of the 15 products in (2.22). Each $f_{i}^{5}$ is the product of 12 eta-functions, and by Theorem 2.10 and a straightforward calculation, each $f_{i}^{5}$ is a modular form of weight 6 on $\Gamma_{1}(200)$ with the multiplier system $v_{5}$, where for $A=\left(\begin{array}{ll}a & b \\ c & d\end{array}\right) \in \Gamma_{1}(200)$, $v_{5}(A)=\left(\frac{10}{|d|}\right) \zeta_{24}^{\frac{1}{100} c\left(a-a d^{2}-d\right)+22 b d+3(d-1)}$. By Theorem 2.12 and a straightforward calculation, each $g_{i}^{5}$ is a modular form of weight 0 on $\Gamma_{1}(200)$ with the multiplier system $I$. Therefore, each $f_{i}^{5} g_{i}^{5}$ is a modular form of weight 6 on $\Gamma_{1}(200)$ with multiplier system $v_{5}$. By Theorem 2.11, $\left[\Gamma(1): \Gamma_{1}(200)\right]=28800$. Let $F_{5}$ denote the difference of the left and right sides of (2.22). Applying Theorem 2.9, for a fundamental region $R$ for $\Gamma_{1}(200)$, we deduce that, for $F_{5}$,

$$
\sum_{z \in R} \operatorname{Ord}_{\Gamma_{1}(200)}\left(F_{5} ; z\right)=\frac{6 \cdot 28800}{12}=14400 \geq \operatorname{ord}\left(F_{5} ; \infty\right)
$$

since both sides of (2.22) are analytic on $R$. Using Mathematica, we calculated the Taylor series of $F_{5}$ about $q=0$ (or about the cusp $z=\infty$ ) and found that 
$F_{5}=O\left(q^{14401}\right)$. Unless $F_{5}$ is a constant, we have a contradiction to $(2.23)$. We have therefore completed the proof of Theorem 2.17.

Theorem 2.18. For $z \in \mathbf{H}$,

$$
\begin{aligned}
\eta_{10}^{8} & \eta_{20}^{3} \eta_{50}^{3} \eta_{10,1} \eta_{20,4}^{2} \eta_{20,6} \eta_{20,8}^{2} \eta_{50,5} \eta_{50,15} \eta_{50,25} \eta_{200,50} \eta_{200,100} \\
& \times\left(\eta_{10,1} \eta_{20,10} \eta_{50,10} \eta_{100,40}-\eta_{10,1} \eta_{20,10} \eta_{50,20} \eta_{100,20}-2 \eta_{10,3} \eta_{20,2} \eta_{50,20} \eta_{100,20}\right) \\
+ & 4 \eta_{10} \eta_{20}^{10} \eta_{50}^{2} \eta_{100} \eta_{10,1} \eta_{10,3} \eta_{20,2} \eta_{20,4}^{2} \eta_{20,6} \eta_{20,8}^{2} \eta_{20,10}^{3} \eta_{40,10} \eta_{50,5} \eta_{50,10} \eta_{50,15} \eta_{50,20} \\
& \times \eta_{100,25}^{2} \eta_{200,100} \\
= & \eta_{10}^{8} \eta_{20}^{3} \eta_{50}^{3} \eta_{50,10} \eta_{50,20} \eta_{200,50} \eta_{200,100} \\
& \times\left(\eta_{10,1}^{3} \eta_{20,6} \eta_{20,8}^{2} \eta_{20,10} \eta_{50,5} \eta_{50,15} \eta_{100,50}+\eta_{10,3}^{3} \eta_{20,2} \eta_{20,4}^{2} \eta_{20,10} \eta_{50,5} \eta_{50,15} \eta_{100,50}\right. \\
& -2 \eta_{10,1} \eta_{10,3} \eta_{20,2} \eta_{20,4} \eta_{20,5}^{2} \eta_{20,6} \eta_{20,8} \eta_{50,5} \eta_{50,25} \eta_{100,30} \\
& -\eta_{10,3}^{3} \eta_{20,2} \eta_{20,4}^{2} \eta_{20,10} \eta_{50,5} \eta_{50,25} \eta_{100,30}-\eta_{10,1}^{3} \eta_{20,6} \eta_{20,8}^{2} \eta_{20,10} \eta_{50,15} \eta_{50,25} \eta_{100,10} \\
& \left.+2 \eta_{10,1} \eta_{10,3} \eta_{20,2} \eta_{20,4} \eta_{20,5}^{2} \eta_{20,6} \eta_{20,8} \eta_{50,15} \eta_{50,25} \eta_{100,10}\right)
\end{aligned}
$$

Proof. For $1 \leq i \leq 10$, let $f_{i}^{6}$ be the product of eta-functions in each of the 10 products in (2.24), and $g_{i}^{6}$ be the product of the generalized eta-functions in each of the 10 products in (2.24). Each $f_{i}^{6}$ is the product of 14 eta-functions, and by Theorem 2.10 and a straightforward calculation, each $f_{i}^{6}$ is a modular form of weight 7 on $\Gamma_{1}(200)$ with the multiplier system $v_{6}$, where for $A=\left(\begin{array}{ll}a & b \\ c & d\end{array}\right) \in \Gamma_{1}(200)$,

$$
v_{6}(A)=\left(\frac{10}{|d|}\right) \zeta_{24}^{\frac{1}{20} c\left(a-a d^{2}-d\right)+2 b d+3(d-1)}
$$

By Theorem 2.12 and a straightforward calculation, each $g_{i}^{6}$ is a modular form of weight 0 on $\Gamma_{1}(200)$ with the multiplier system $I$. Therefore, each $f_{i}^{6} g_{i}^{6}$ is a modular form of weight 7 on $\Gamma_{1}(200)$ with multiplier system $v_{6}$. Let $F_{6}$ denote the difference of the left and right sides of (2.24). Applying Theorem 2.9, for a fundamental region $R$ for $\Gamma_{1}(200)$, we deduce that, for $F_{6}$,

$$
\sum_{z \in R} \operatorname{Ord}_{\Gamma_{1}(200)}\left(F_{6} ; z\right)=\frac{7 \cdot 28800}{12}=16800 \geq \operatorname{ord}\left(F_{6} ; \infty\right)
$$

since both sides of (2.24) are analytic on $R$. Using Mathematica, we calculated the Taylor series of $F_{6}$ about $q=0$ (or about the cusp $z=\infty$ ) and found that $F_{6}=O\left(q^{16801}\right)$. Unless $F_{6}$ is a constant, we have a contradiction to $(2.25)$. We have thus completed the proof of Theorem 2.18.

We have proved six eta-function identities with the properties of modular forms. In the next step, we will derive six theta function identities from the eta-function identities above. 
Theorem 2.19. For $|q|<1$,

$$
\begin{aligned}
& -\frac{f\left(-q^{5},-q^{45}\right) f\left(-q^{40},-q^{60}\right) f\left(-q^{50},-q^{50}\right)}{f\left(-q^{20},-q^{30}\right)^{2}}\left(q^{6} f\left(-q^{10},-q^{40}\right)-q^{4} f\left(-q^{20},-q^{30}\right)\right) \\
& -\frac{f\left(-q^{50},-q^{50}\right)}{f\left(-q^{10},-q^{40}\right)}\left(q^{3} f\left(-q^{15},-q^{35}\right) f\left(-q^{20},-q^{80}\right)-2 q^{2} f\left(-q^{5},-q^{45}\right) f\left(-q^{40},-q^{60}\right)\right) \\
& -2 q \frac{f\left(-q^{5},-q^{45}\right)^{2} f\left(-q^{40},-q^{60}\right)\left(q^{50} ; q^{50}\right)_{\infty}\left(q^{100} ; q^{100}\right)_{\infty}}{f\left(-q^{15},-q^{35}\right) f\left(-q^{10},-q^{90}\right) f\left(-q^{20},-q^{80}\right)} \\
& +\frac{f\left(-q^{5},-q^{45}\right)^{2} f\left(-q^{40},-q^{60}\right)\left(q^{50} ; q^{50}\right)^{3}}{f\left(-q^{25},-q^{25}\right) f\left(-q^{10},-q^{90}\right) f\left(-q^{20},-q^{80}\right) f\left(-q^{30},-q^{70}\right)} \\
& =\frac{f\left(-q,-q^{9}\right)^{2} f\left(-q^{8},-q^{12}\right)\left(q^{10} ; q^{10}\right)_{\infty}\left(q^{20} ; q^{20}\right)_{\infty}}{f\left(-q^{3},-q^{7}\right) f\left(-q^{2},-q^{18}\right) f\left(-q^{4},-q^{16}\right)} .
\end{aligned}
$$

Proof. By applying $\eta_{100,50} \eta_{100}^{2}=\eta_{50}^{2}$ to the right side of (2.14), dividing both sides of $(2.14)$ by $q^{\frac{451}{30}} \eta_{50}^{18} \eta_{10,2}^{4} \eta_{10,3} \eta_{20,2} \eta_{20,4} \eta_{50,10} \eta_{50,15} \eta_{50,20}^{2} \eta_{50,25} / \eta_{100}^{2}$, and applying (2.12) and (2.13), we derive (2.26) from (2.14).

Theorem 2.20. For $|q|<1$,

$$
\begin{aligned}
& \frac{f\left(-q^{15},-q^{35}\right) f\left(-q^{20},-q^{80}\right) f\left(-q^{50},-q^{50}\right)}{f\left(-q^{10},-q^{40}\right)^{2}}\left(q^{2} f\left(-q^{10},-q^{40}\right)+f\left(-q^{20},-q^{30}\right)\right) \\
& -\frac{f\left(-q^{50},-q^{50}\right)}{f\left(-q^{20},-q^{30}\right)}\left(q^{3} f\left(-q^{5},-q^{45}\right) f\left(-q^{40},-q^{60}\right)+2 q^{4} f\left(-q^{15},-q^{35}\right) f\left(-q^{20},-q^{80}\right)\right) \\
& -2 q^{5} \frac{f\left(-q^{15},-q^{35}\right)^{2} f\left(-q^{20},-q^{80}\right)\left(q^{50} ; q^{50}\right)_{\infty}\left(q^{100} ; q^{100}\right)_{\infty}}{f\left(-q^{5},-q^{45}\right) f\left(-q^{30},-q^{70}\right) f\left(-q^{40},-q^{60}\right)} \\
& +q \frac{f\left(-q^{15},-q^{35}\right)^{2} f\left(-q^{20},-q^{80}\right)\left(q^{50} ; q^{50}\right)^{3}}{f\left(-q^{25},-q^{25}\right) f\left(-q^{10},-q^{90}\right) f\left(-q^{30},-q^{70}\right) f\left(-q^{40},-q^{60}\right)} \\
& =\frac{f\left(-q^{3},-q^{7}\right)^{2} f\left(-q^{4},-q^{16}\right)\left(q^{10} ; q^{10}\right)_{\infty}\left(q^{20} ; q^{20}\right)_{\infty}}{f\left(-q,-q^{9}\right) f\left(-q^{6},-q^{14}\right) f\left(-q^{8},-q^{12}\right)} .
\end{aligned}
$$

Proof. By applying $\eta_{100,50} \eta_{100}^{2}=\eta_{50}^{2}$ to the right side of (2.16), dividing both sides of $(2.16)$ by $q^{\frac{589}{30}} \eta_{50}^{18} \eta_{10,1} \eta_{10,2}^{16} \eta_{20,6} \eta_{20,8} \eta_{50,5} \eta_{50,10}^{2} \eta_{50,20} \eta_{50,25} / \eta_{100}^{2}$, and applying (2.12) and (2.13), we derive (2.27) from (2.16).

Theorem 2.21. If $|q|<1$, then

$$
\begin{aligned}
& q^{5} \frac{f\left(-q^{5},-q^{45}\right) f\left(-q^{10},-q^{40}\right) f\left(-q^{40},-q^{60}\right) f\left(-q^{50},-q^{50}\right)}{f\left(-q^{20},-q^{30}\right)^{2}} \\
& +\frac{f\left(-q^{15},-q^{35}\right) f\left(-q^{20},-q^{30}\right) f\left(-q^{20},-q^{80}\right) f\left(-q^{50},-q^{50}\right)}{f\left(-q^{10},-q^{40}\right)^{2}}-3 q^{5} f\left(-q^{25},-q^{75}\right)^{2} \\
& =f\left(-q^{5},-q^{15}\right)^{2} .
\end{aligned}
$$

Proof. By applying $\eta_{25,5} \eta_{25,10} \eta_{25}=\eta_{5}$ to the right side of (2.18) in Theorem 2.15, dividing both sides of (2.18) by $q^{\frac{25}{4}} \eta_{5}^{2} \eta_{5,1}^{10} \eta_{6,1}^{3} \eta_{50,10}^{2} \eta_{50,20}^{2}$, and applying (2.12) and (2.13), we derive (2.28) from (2.18). 
Theorem 2.22. For $|q|<1$,

$$
\begin{aligned}
& f\left(q^{25}, q^{25}\right) f\left(-q^{25},-q^{25}\right)+q^{2} f\left(q^{15}, q^{35}\right) f\left(-q^{15},-q^{35}\right)-q^{8} f\left(q^{5}, q^{45}\right) f\left(-q^{5},-q^{45}\right) \\
& -q^{4} f\left(q^{25}, q^{25}\right) f\left(-q^{5},-q^{45}\right)-q f\left(q^{15}, q^{35}\right) f\left(-q^{25},-q^{25}\right) \\
& +2 q^{5} f\left(q^{15}, q^{35}\right) f\left(-q^{5},-q^{45}\right)-q^{5} f\left(q^{5}, q^{45}\right) f\left(-q^{15},-q^{35}\right) \\
& =\frac{f\left(-q,-q^{9}\right)\left(q^{4} ; q^{4}\right)_{\infty}\left(q^{10} ; q^{10}\right)_{\infty}^{2}}{f\left(-q^{3},-q^{7}\right) f\left(-q^{2},-q^{18}\right)} .
\end{aligned}
$$

Proof. We can easily derive that, for any integers $n$,

$$
\frac{\left(q^{50} ; q^{50}\right)_{\infty}^{2} f\left(-q^{2 n},-q^{100-2 n}\right)}{\left(q^{100} ; q^{100}\right)_{\infty} f\left(-q^{n},-q^{50-n}\right)}=f\left(q^{n}, q^{50-n}\right) .
$$

Now, applying $\eta_{20} \eta_{20,4} \eta_{20,8}=\eta_{4}$ to the right side of (2.20), dividing both sides of (2.20) by $q^{\frac{329}{30}} \eta_{4}^{2} \eta_{10,1}^{2} \eta_{10,2}^{3} \eta_{10,3} \eta_{20,2} \eta_{50,5} \eta_{50,15} \eta_{50,25}$, applying $(2.12),(2.13)$, and (2.30) with $n$ replaced by 5,15 and 25 , repeatedly, we complete the proof.

Theorem 2.23. For $|q|<1$,

$$
\begin{aligned}
& f\left(q^{20}, q^{30}\right)\left(\frac{f\left(-q,-q^{9}\right)\left(q^{4} ; q^{4}\right)_{\infty}\left(q^{10} ; q^{10}\right)_{\infty}^{2}}{f\left(-q^{3},-q^{7}\right) f\left(-q^{2},-q^{18}\right)}-4 q f\left(-q^{4},-q^{16}\right) f\left(-q^{8},-q^{12}\right)\right) \\
& +q^{2} f\left(q^{10}, q^{40}\right)\left(\frac{f\left(-q,-q^{9}\right)\left(q^{4} ; q^{4}\right)_{\infty}\left(q^{10} ; q^{10}\right)_{\infty}^{2}}{f\left(-q^{3},-q^{7}\right) f\left(-q^{2},-q^{18}\right)}+6 q f\left(-q^{4},-q^{16}\right) f\left(-q^{8},-q^{12}\right)\right) \\
& -4 q^{6} f\left(q^{50}, q^{150}\right)\left(\frac{f\left(-q,-q^{9}\right)\left(q^{4} ; q^{4}\right)_{\infty}\left(q^{10} ; q^{10}\right)_{\infty}^{2}}{f\left(-q^{3},-q^{7}\right) f\left(-q^{2},-q^{18}\right)}+q f\left(-q^{4},-q^{16}\right) f\left(-q^{8},-q^{12}\right)\right) \\
& =f\left(q^{25}, q^{25}\right)\left(\frac{f\left(-q,-q^{9}\right)^{2} f\left(-q^{8},-q^{12}\right)\left(q^{10} ; q^{10}\right)_{\infty}\left(q^{20} ; q^{20}\right)_{\infty}}{f\left(-q^{3},-q^{7}\right) f\left(-q^{2},-q^{18}\right) f\left(-q^{4},-q^{16}\right)}\right. \\
& \left.-4 q f\left(-q^{5},-q^{15}\right)^{2}-q^{3} \frac{f\left(-q^{3},-q^{7}\right)^{2} f\left(-q^{4},-q^{16}\right)\left(q^{10} ; q^{10}\right)_{\infty}\left(q^{20} ; q^{20}\right)_{\infty}}{f\left(-q,-q^{9}\right) f\left(-q^{6},-q^{14}\right) f\left(-q^{8},-q^{12}\right)}\right) \\
& +q f\left(q^{15}, q^{35}\right)\left(2 \frac{f\left(-q,-q^{9}\right)^{2} f\left(-q^{8},-q^{12}\right)\left(q^{10} ; q^{10}\right)_{\infty}\left(q^{20} ; q^{20}\right)_{\infty}}{f\left(-q^{3},-q^{7}\right) f\left(-q^{2},-q^{18}\right) f\left(-q^{4},-q^{16}\right)}\right. \\
& \left.+2 q f\left(-q^{5},-q^{15}\right)^{2}+3 q \frac{f\left(-q^{3},-q^{7}\right)^{2} f\left(-q^{4},-q^{16}\right)\left(q^{10} ; q^{10}\right)_{\infty}\left(q^{20} ; q^{20}\right)_{\infty}}{f\left(-q,-q^{9}\right) f\left(-q^{6},-q^{14}\right) f\left(-q^{8},-q^{12}\right)}\right) \\
& -q^{4} f\left(q^{25}, q^{25}\right)\left(3 \frac{f\left(-q,-q^{9}\right)^{2} f\left(-q^{8},-q^{12}\right)\left(q^{10} ; q^{10}\right)_{\infty}\left(q^{20} ; q^{20}\right)_{\infty}}{f\left(-q^{3},-q^{7}\right) f\left(-q^{2},-q^{18}\right) f\left(-q^{4},-q^{16}\right)}\right. \\
& \left.-2 q f\left(-q^{5},-q^{15}\right)^{2}+2 q \frac{f\left(-q^{3},-q^{7}\right)^{2} f\left(-q^{4},-q^{16}\right)\left(q^{10} ; q^{10}\right)_{\infty}\left(q^{20} ; q^{20}\right)_{\infty}}{f\left(-q,-q^{9}\right) f\left(-q^{6},-q^{14}\right) f\left(-q^{8},-q^{12}\right)}\right) .
\end{aligned}
$$

Proof. Applying $\eta_{20}^{2} \eta_{20,10}=\eta_{10}^{2}, \eta_{20} \eta_{40,10}=\eta_{10}, \eta_{20} \eta_{20,4} \eta_{20,8}=\eta_{4}$, and $\eta_{50} \eta_{50,10} \times$ $\eta_{50,20}=\eta_{10}$ to $(2.22)$, dividing both sides of $(2.22)$ by $q^{\frac{233}{6}} \eta_{10}^{8} \eta_{20} \eta_{10,1} \eta_{10,3} \eta_{20,2} \eta_{20,4} \times$ $\eta_{20,6} \eta_{20,8} \eta_{50,5} \eta_{50,10} \eta_{50,15} \eta_{50,20} \eta_{50,25} \eta_{200,50} \eta_{200,100}$, and applying $(2.12)$, $(2.13)$, and (2.30) with $n$ replaced by $5,10,15,20$ and 25 , repeatedly, and with $q$ and $n$ replaced by $q^{4}$ and $q^{50}$, respectively, we derive (2.32) from (2.22). 
Theorem 2.24.

$(2.32)$

$$
\begin{aligned}
& f\left(q^{20}, q^{30}\right) \frac{f\left(-q,-q^{9}\right)\left(q^{4} ; q^{4}\right)_{\infty}\left(q^{10} ; q^{10}\right)_{\infty}^{2}}{f\left(-q^{3},-q^{7}\right) f\left(-q^{2},-q^{18}\right)} \\
& -q^{2} f\left(q^{10}, q^{40}\right)\left(\frac{f\left(-q,-q^{9}\right)\left(q^{4} ; q^{4}\right)_{\infty}\left(q^{10} ; q^{10}\right)_{\infty}^{2}}{f\left(-q^{3},-q^{7}\right) f\left(-q^{2},-q^{18}\right)}+2 q f\left(-q^{4},-q^{16}\right) f\left(-q^{8},-q^{12}\right)\right) \\
& +4 q^{7} f\left(q^{50}, q^{150}\right) f\left(-q^{4},-q^{16}\right) f\left(-q^{8},-q^{12}\right) \\
& =f\left(q^{25}, q^{25}\right)\left(\frac{f\left(-q,-q^{9}\right)^{2} f\left(-q^{8},-q^{12}\right)\left(q^{10} ; q^{10}\right)_{\infty}\left(q^{20} ; q^{20}\right)_{\infty}}{f\left(-q^{3},-q^{7}\right) f\left(-q^{2},-q^{18}\right) f\left(-q^{4},-q^{16}\right)}\right. \\
& \left.\quad+q \frac{f\left(-q^{3},-q^{7}\right)^{2} f\left(-q^{4},-q^{16}\right)\left(q^{10} ; q^{10}\right)_{\infty}\left(q^{20} ; q^{20}\right)_{\infty}}{f\left(-q,-q^{9}\right) f\left(-q^{6},-q^{14}\right) f\left(-q^{8},-q^{12}\right)}\right) \\
& -q^{2} f\left(q^{15}, q^{35}\right)\left(2 f\left(-q^{5},-q^{15}\right)^{2}\right. \\
& \left.\quad+3 q \frac{f\left(-q^{3},-q^{7}\right)^{2} f\left(-q^{4},-q^{16}\right)\left(q^{10} ; q^{10}\right)_{\infty}\left(q^{20} ; q^{20}\right)_{\infty}}{f\left(-q,-q^{9}\right) f\left(-q^{6},-q^{14}\right) f\left(-q^{8},-q^{12}\right)}\right) \\
& -q^{4} f\left(q^{5}, q^{45}\right)\left(\frac{f\left(-q,-q^{9}\right)^{2} f\left(-q^{8},-q^{12}\right)\left(q^{10} ; q^{10}\right)_{\infty}\left(q^{20} ; q^{20}\right)_{\infty}}{f\left(-q^{3},-q^{7}\right) f\left(-q^{2},-q^{18}\right) f\left(-q^{4},-q^{16}\right)}\right. \\
& \left.\quad-2 q f\left(-q^{5},-q^{15}\right)^{2}\right) .
\end{aligned}
$$

Proof. Applying $\eta_{20}^{2} \eta_{20,10}=\eta_{10}^{2}, \eta_{20} \eta_{40,10}=\eta_{10}, \eta_{20} \eta_{20,4} \eta_{20,8}=\eta_{4}$, and $\eta_{50} \eta_{100,25}$ $=\eta_{25}$ to (2.24), dividing both sides of (2.24) by $q^{\frac{233}{6}} \eta_{10}^{8} \eta_{20} \eta_{50}^{2} \eta_{10,1} \eta_{10,3} \eta_{20,2} \eta_{20,4} \times$ $\eta_{20,6} \eta_{20,8} \eta_{50,5} \eta_{50,10} \eta_{50,15} \eta_{50,20} \eta_{50,25} \eta_{200,50} \eta_{200,100}$, and applying (2.12), (2.13), and (2.30) with $n$ replaced by $5,10,15,20$ and 25, repeatedly, and with $q$ and $n$ replaced by $q^{4}$ and $q^{50}$, respectively, we derive (2.32) from (2.24).

\section{Proof of Ramanujan's Seventh identity}

In this section, we will prove Ramanujan's seventh identity, (1.1).

Theorem 3.1 (Ramanujan's seventh tenth order mock theta function identity).

$$
\begin{aligned}
& \int_{0}^{\infty} \frac{e^{-\pi n x^{2}}}{\cosh \frac{2 \pi x}{\sqrt{5}}+\frac{1+\sqrt{5}}{4}} d x+\frac{1}{\sqrt{n}} e^{\frac{\pi}{5 n}} \psi\left(-e^{-\frac{\pi}{n}}\right) \\
& =\sqrt{\frac{5+\sqrt{5}}{2}} e^{-\frac{\pi n}{5}} \phi\left(-e^{-\pi n}\right)-\frac{\sqrt{5}+1}{2 \sqrt{n}} e^{-\frac{\pi}{5 n}} \phi\left(-e^{-\frac{\pi}{n}}\right) .
\end{aligned}
$$

Proof. Replacing $\omega, x, t$, and $\theta$ by $5 i n,-5 n, \frac{z}{\sqrt{5}}+1$, and $\theta^{\prime}-i$, respectively, in the left side of (1.14), we find that

$$
\int_{-\infty}^{\infty} \frac{e^{\pi i \omega t^{2}-2 \pi x t}}{e^{2 \pi t}-e^{2 \pi i \theta}} d t=\frac{e^{5 \pi n-2 \pi}}{\sqrt{5}} \int_{-\infty}^{\infty} \frac{e^{-\pi n z^{2}}}{e^{\frac{2 \pi z}{\sqrt{5}}}-e^{2 \pi i \theta^{\prime}}} d z
$$

Replacing $\theta^{\prime}$ by $\frac{2}{5}$ in (3.1), and using (1.14), we have

$$
\int_{-\infty}^{\infty} \frac{e^{-\pi n z^{2}}}{e^{\frac{2 \pi z}{\sqrt{5}}}+e^{-\frac{\pi i}{5}}} d z=\sqrt{5} e^{\frac{4 \pi n}{5}-\frac{4 \pi i}{5}} \frac{F\left(\frac{2}{5}, \frac{i}{5 n}\right)-5 n F(2 i n, 5 i n)}{5 i n \theta_{11}(2 i n, 5 i n)}
$$


and replacing $\theta^{\prime}$ by $\frac{3}{5}$ in (3.1), and using (1.14), we have

$$
\int_{-\infty}^{\infty} \frac{e^{-\pi n z^{2}}}{e^{\frac{2 \pi z}{\sqrt{5}}}+e^{\frac{\pi i}{5}}} d z=\sqrt{5} e^{\frac{9 \pi n}{5}-\frac{6 \pi i}{5}} \frac{F\left(\frac{3}{5}, \frac{i}{5 n}\right)-5 n F(3 i n, 5 i n)}{5 i n \theta_{11}(3 i n, 5 i n)} .
$$

Using (3.2) and (3.3), we can easily derive that

$$
\begin{gathered}
\int_{0}^{\infty} \frac{e^{-\pi n x^{2}}}{\cosh \frac{2 \pi x}{\sqrt{5}}+\frac{1+\sqrt{5}}{4}} d x=\frac{1}{2} \int_{-\infty}^{\infty} \frac{e^{-\pi n x^{2}}}{\cosh \frac{2 \pi x}{\sqrt{5}}+\frac{1+\sqrt{5}}{4}} d x \\
=-\frac{1}{2} \int_{-\infty}^{\infty} \frac{e^{-\pi n x^{2}}}{i \sin \frac{\pi}{5}}\left(\frac{1}{1+e^{\frac{2 \pi x}{\sqrt{5}}+\frac{\pi}{5} i}}-\frac{1}{1+e^{\frac{2 \pi x}{\sqrt{5}}-\frac{\pi}{5} i}}\right) d x \\
=-\frac{1}{2 i \sin \frac{\pi}{5}}\left(e^{-\frac{\pi}{5} i} \int_{-\infty}^{\infty} \frac{e^{-\pi n x^{2}}}{e^{\frac{2 \pi x}{\sqrt{5}}}+e^{-\frac{\pi}{5} i}} d x-e^{\frac{\pi}{5} i} \int_{-\infty}^{\infty} \frac{e^{-\pi n x^{2}}}{e^{\frac{2 \pi x}{\sqrt{5}}}+e^{\frac{\pi}{5} i}} d x\right) \\
=-\frac{\sqrt{5+\sqrt{5}}}{5 n \sqrt{2}}\left(e^{-\frac{\pi n}{5}+\pi n} \frac{F\left(\frac{2}{5}, \frac{i}{5 n}\right)-5 n F(2 i n, 5 i n)}{\theta_{11}(2 i n, 5 i n)}\right. \\
\left.-e^{-\frac{\pi n}{5}+2 \pi n} \frac{F\left(\frac{3}{5}, \frac{i}{5 n}\right)-5 n F(3 i n, 5 i n)}{\theta_{11}(3 i n, 5 i n)}\right) .
\end{gathered}
$$

Let $q=e^{-\pi n}$. Then, using (1.15), (1.16), (1.3), (1.10) with $q$ and $z$ replaced by $-q$ and $q^{4}$, respectively, (1.8), (1.11) with $q, z$, and $x$ replaced by $-q^{5}, 1$ and $q^{2}$, respectively, (1.13) with $q$ replaced by $-q$, and Theorem 2.4, we can derive that

$$
\begin{aligned}
& \sqrt{\frac{5+\sqrt{5}}{2}}\left(e^{-\frac{\pi n}{5}+\pi n} \frac{F(2 i n, 5 i n)}{\theta_{11}(2 i n, 5 i n)}-e^{-\frac{\pi n}{5}+2 \pi n} \frac{F(3 i n, 5 i n)}{\theta_{11}(3 i n, 5 i n)}\right) \\
& =-\sqrt{\frac{5+\sqrt{5}}{2}} e^{-\frac{\pi n}{5}}\left(\frac{\sum_{m} \frac{(-1)^{m} q^{5 m^{2}+9 m+3}}{1+q^{10 m+5}}}{f\left(-q^{4},-q^{6}\right)}+\frac{\sum_{m} \frac{(-1)^{m} q^{5 m^{2}+9 m+3}}{1+q^{10 m+5}}}{f\left(-q^{4},-q^{6}\right)}\right) \\
& =\sqrt{\frac{5+\sqrt{5}}{2}} e^{-\frac{\pi n}{5}} \frac{L_{2}\left(q, q^{4}\right)}{f\left(-q^{4},-q^{6}\right)} \\
& =\sqrt{\frac{5+\sqrt{5}}{2}} e^{-\frac{\pi n}{5}}\left(\phi(-q)-\frac{\left(-q^{5} ;-q^{5}\right)_{\infty}\left(q^{10} ; q^{10}\right)_{\infty} f\left(-q^{4},-q^{6}\right)}{f\left(-q^{2}, q^{3}\right) f\left(-q^{2},-q^{8}\right)}\right. \\
& \left.\quad+2 q \frac{\left(-q^{5} ;-q^{5}\right)_{\infty}^{2}\left(q^{10} ; q^{10}\right)_{\infty}^{2}}{f\left(q^{5}, q^{5}\right)^{2} f\left(-q^{4},-q^{6}\right)}\right) \\
& =\sqrt{\frac{5+\sqrt{5}}{2}} e^{-\frac{\pi n}{5}} \phi(-q)-\sqrt{\frac{5+\sqrt{5}}{2}} e^{-\frac{\pi n}{5}} \frac{f\left(-q,-q^{9}\right) f\left(q^{3}, q^{7}\right) f\left(q^{4}, q^{6}\right)}{f\left(-q^{4},-q^{6}\right) f\left(q, q^{9}\right)} .
\end{aligned}
$$

We now need to use the following transformation formula for $\theta_{11}$ in [ML, p. 330]:

$$
\theta_{11}\left(\frac{x}{\omega},-\frac{1}{\omega}\right)=-i \sqrt{-i \omega} e^{\pi i x^{2} / \omega} \theta_{11}(x, \omega) .
$$

Replacing $x$ and $\omega$ by 2 in and $5 i n$, respectively, in (3.6), we find that

$$
\theta_{11}\left(\frac{2}{5}, \frac{i}{5 n}\right)=-i \sqrt{5 n} e^{-\frac{4 n \pi}{5}} \theta_{11}(2 i n, 5 i n),
$$


and replacing $x$ and $\omega$ by 3 in and 5in, respectively, in (3.6), we find that

$$
\theta_{11}\left(\frac{3}{5}, \frac{i}{5 n}\right)=-i \sqrt{5 n} e^{-\frac{9 n \pi}{5}} \theta_{11}(3 i n, 5 i n) .
$$

Let $q_{1}=e^{-\frac{\pi}{5 n}}, \alpha=e^{\frac{2 \pi i}{5}}$, and $\beta=e^{\frac{3 \pi i}{5}}=-\alpha^{-1}$. Then, using (3.7), (3.8), (1.15), and (1.16), we find that

$$
\begin{aligned}
\frac{i}{\sqrt{5 n}}\left(e^{\frac{4 \pi n}{5}} \frac{F\left(\frac{2}{5}, \frac{i}{5 n}\right)}{\theta_{11}(2 i n, 5 i n)}-e^{\frac{9 \pi n}{5}}\right. & \left.\frac{F\left(\frac{3}{5}, \frac{i}{5 n}\right)}{\theta_{11}(3 i n, 5 i n)}\right)=\frac{F\left(\frac{2}{5}, \frac{i}{5 n}\right)}{\theta_{11}\left(\frac{2}{5}, \frac{i}{5 n}\right)}-\frac{F\left(\frac{3}{5}, \frac{i}{5 n}\right)}{\theta_{11}\left(\frac{3}{5}, \frac{i}{5 n}\right)} \\
& =\frac{\sum_{m} \frac{(-1)^{m} q_{1}^{m^{2}+m} \alpha^{2 m}\left(1-q_{1}^{2 m+1}\right)}{1+q_{1}^{2 m+1}}}{\sum_{m}(-1)^{m} q_{1}^{m^{2}+m} \alpha^{2 m}} .
\end{aligned}
$$

By (1.3), we easily reformulate the denominator of the right hand side of (3.9):

$$
\begin{aligned}
\sum_{m}(-1)^{m} q_{1}^{m^{2}+m} \alpha^{2 m} & =\sum_{l=0}^{4} \alpha^{2 l} \sum_{m}(-1)^{m+l} q_{1}^{25 m^{2}+10 m l+l^{2}+5 m+l} \\
& =\left(1-\alpha^{8}\right) f\left(-q_{1}^{20},-q_{1}^{30}\right)-q_{1}^{2}\left(\alpha^{2}-\alpha^{6}\right) f\left(-q_{1}^{10},-q_{1}^{40}\right) .
\end{aligned}
$$

Now, multiplying by $1-q_{1}^{2 m+1}+q_{1}^{4 m+2}-q_{1}^{6 m+3}+q_{1}^{8 m+4}$ both the numerator and the denominator of the numerator of the right side of (3.9), we find that the numerator of the right side of (3.9) equals

$$
\begin{aligned}
& \sum_{m} \frac{(-1)^{m} q_{1}^{m^{2}+m} \alpha^{2 m}}{1+q_{1}^{10 m+5}}\left\{1-2\left(q_{1}^{2 m+1}-q_{1}^{4 m+2}+q_{1}^{6 m+3}-q_{1}^{8 m+4}\right)-q_{1}^{10 m+5}\right\} \\
& =\sum_{l=0}^{4} \sum_{m} \frac{(-1)^{m+l} q_{1}^{(5 m+l)^{2}+(5 m+l)} \alpha^{10 m+2 l}}{1+q_{1}^{50 m+10 l+5}} \\
& \quad \times\left\{1-2\left(q_{1}^{10 m+2 l+1}-q_{1}^{20 m+4 l+2}+q_{1}^{30 m+6 l+3}-q_{1}^{40 m+8 l+4}\right)-q_{1}^{50 m+10 l+5}\right\} .
\end{aligned}
$$

We will transform some of the generalized Lambert series in (3.11) into $L_{1}$ and $L_{2}$, and we need the following hypergeometric series from [GR, p. 128] to handle the other generalized Lambert series in (3.11):

$$
\begin{aligned}
& \sum_{m} \frac{\left(q a^{1 / 2} ; q\right)_{n}\left(-q a^{1 / 2} ; q\right)_{n}(b ; q)_{n}(c ; q)_{n}(d ; q)_{n}(e ; q)_{n}}{\left(a^{1 / 2} ; q\right)_{n}\left(-a^{1 / 2} ; q\right)_{n}(a q / b ; q)_{n}(a q / c ; q)_{n}(a q / d ; q)_{n}(a q / e ; q)_{n}}\left(\frac{q a^{2}}{b c d e}\right) \\
& =\frac{(a q ; q)_{\infty}(a q / b c ; q)_{\infty}(a q / b d ; q)_{\infty}(a q / b e ; q)_{\infty}(a q / c d ; q)_{\infty}(a q / c e ; q)_{\infty}}{(a q / b ; q)_{\infty}(a q / c ; q)_{\infty}(a q / d ; q)_{\infty}(a q / e ; q)_{\infty}(q / b ; q)_{\infty}(q / c ; q)_{\infty}(q / d ; q)_{\infty}} \\
& \quad \times \frac{(a q / d e ; q)_{\infty}(q ; q)_{\infty}(q / a ; q)_{\infty}}{(q / e ; q)_{\infty}\left(q a^{2} / b c d e ; q\right)_{\infty}}
\end{aligned}
$$

provided $\left|q a^{2} / b c d e\right|<1$.

Replacing $q, a, b$, and $c$ by $-q_{1}^{25}, q_{1}^{5}, \sqrt{q_{1}^{5}} i$ and $-\sqrt{q_{1}^{5}} i$, respectively, in (3.12), letting $d$ and $e$ tend to $\infty$, and using (1.3), (1.5), and (2.30) with $n$ replaced by 5 , 
we find that

$$
\begin{array}{r}
\sum_{m}(-1)^{m} q_{1}^{25 m^{2}+5 m} \frac{1-q_{1}^{50 m+5}}{1+q_{1}^{50 m+5}}=\frac{f\left(-q_{1}^{5}, q_{1}^{20}\right)\left(-q_{1}^{25} ;-q_{1}^{25}\right)_{\infty}\left(q_{1}^{50} ; q_{1}^{50}\right)_{\infty}}{f\left(q_{1}^{5}, q_{1}^{45}\right)} \\
=\frac{f\left(-q_{1}^{5},-q_{1}^{45}\right)^{2} f\left(-q_{1}^{40},-q_{1}^{60}\right) f\left(-q_{1}^{50},-q_{1}^{50}\right)\left(q_{1}^{100} ; q_{1}^{100}\right)_{\infty}^{3}}{f\left(-q_{1}^{10},-q_{1}^{90}\right) f\left(-q_{1}^{20},-q_{1}^{80}\right) f\left(-q_{1}^{30},-q_{1}^{70}\right) f\left(-q_{1}^{25},-q_{1}^{75}\right)^{2}} .
\end{array}
$$

Replacing $q, a, b$, and $c$ by $-q_{1}^{25}, q_{1}^{15}, \sqrt{q_{1}^{15}} i$ and $-\sqrt{q_{1}^{15}} i$, respectively, in (3.12), letting $d$ and $e$ tend to $\infty$, and using (1.3), (1.5), and (2.30) with $n$ replaced by 15, we find that

$$
\begin{gathered}
\sum_{m}(-1)^{m} q_{1}^{25 m^{2}+15 m} \frac{1-q_{1}^{50 m+15}}{1+q_{1}^{50 m+15}}=\frac{f\left(-q_{1}^{15}, q_{1}^{10}\right)\left(-q_{1}^{25} ;-q_{1}^{25}\right)_{\infty}\left(q_{1}^{50} ; q_{1}^{50}\right)_{\infty}}{f\left(q_{1}^{15}, q_{1}^{35}\right)} \\
=\frac{f\left(-q_{1}^{15},-q_{1}^{35}\right)^{2} f\left(-q_{1}^{20},-q_{1}^{80}\right) f\left(-q_{1}^{50},-q_{1}^{50}\right)\left(q_{1}^{100} ; q_{1}^{100}\right)_{\infty}^{3}}{f\left(-q_{1}^{10},-q_{1}^{90}\right) f\left(-q_{1}^{30},-q_{1}^{70}\right) f\left(-q_{1}^{40},-q_{1}^{60}\right) f\left(-q_{1}^{25},-q_{1}^{75}\right)^{2}} .
\end{gathered}
$$

With Entry 8(vii) in [B1, p. 114], we find that

$$
\sum_{m} \frac{(-1)^{m} q_{1}^{25 m^{2}+15 m}}{1+q_{1}^{50 m+15}}=f\left(-q_{1}^{25},-q_{1}^{75}\right)^{2} .
$$

Using (1.9), (1.10), (3.11), (3.13), (3.14), and (3.15), we find that

$$
\begin{aligned}
& \sum_{m} \frac{(-1)^{m} q_{1}^{m^{2}+m} \alpha^{2 m}\left(1-q_{1}^{2 m+1}\right)}{1+q_{1}^{2 m+1}} \\
& =\left(1+\alpha^{8}\right)\left(q_{1} L_{1}\left(q_{1}^{5}, q_{1}^{40}\right)-q_{1}^{7} L_{2}\left(q_{1}^{5}, 1\right)-q^{-1} L_{1}\left(q^{5}, q^{30}\right)-q^{3} L_{2}\left(q^{5}, q^{40}\right)\right) \\
& \quad+\left(\alpha^{2}+\alpha^{6}\right)\left(q^{5} L_{1}\left(q^{5}, 1\right)+q^{3} L_{2}\left(q^{5}, q^{10}\right)+q^{-1} L_{1}\left(q^{5}, q^{20}\right)+q L_{2}\left(q^{5}, q^{30}\right)\right) \\
& \quad-2 \alpha^{4}\left(q L_{1}\left(q^{5}, q^{10}\right)+q L_{2}\left(q^{5}, q^{20}\right)\right) \\
& \quad+\left(1+\alpha^{8}\right) \frac{f\left(-q_{1}^{5}, q_{1}^{20}\right)\left(-q_{1}^{25} ;-q_{1}^{25}\right)_{\infty}\left(q_{1}^{50} ; q_{1}^{50}\right)_{\infty}}{f\left(q_{1}^{5}, q_{1}^{45}\right)} \\
& \quad-\left(\alpha^{2}+\alpha^{6}\right) q^{2} \frac{f\left(-q_{1}^{15}, q_{1}^{10}\right)\left(-q_{1}^{25} ;-q_{1}^{25}\right)_{\infty}\left(q_{1}^{50} ; q_{1}^{50}\right)_{\infty}}{f\left(q_{1}^{15}, q_{1}^{35}\right)}-2 \alpha^{4} q^{6} f\left(-q_{1}^{25},-q_{1}^{75}\right)^{2} .
\end{aligned}
$$

To proceed to the next step, we need the following result, which can be proved by a the simple calculation:

$$
\left\{\begin{array}{l}
1-2 \alpha^{4}+\alpha^{8}=i \sqrt{\frac{5-\sqrt{5}}{2}}\left(-\alpha^{2}+\alpha^{6}\right) \\
-1+\alpha^{2}+\alpha^{6}-\alpha^{8}=i \sqrt{\frac{5-\sqrt{5}}{5}}\left(1-\alpha^{8}\right) \text { or } i \sqrt{\frac{5+\sqrt{5}}{2}}\left(-\alpha^{2}+\alpha^{6}\right), \\
\alpha^{2}-2 \alpha^{4}+\alpha^{6}=i \sqrt{\frac{5+\sqrt{5}}{2}}\left(1-\alpha^{8}\right) .
\end{array}\right.
$$

Transforming $L_{1}$ and $L_{2}$ in the right side of (3.16) into the sums of $\psi(q)$ and theta functions, and $\phi(q)$ and theta functions, respectively, with (1.7) and (1.8), using (3.10) and (3.17) to simplify the terms related to $\phi(q)$ and $\psi(q)$, using (1.11), (1.12), and (1.13), replacing $q$ by $q_{1}$ in Theorem 2.19, Theorem 2.20, and Theorem 2.21, 
and applying Theorem 2.19, Theorem 2.20, and Theorem 2.21, we find that the right side of (3.16) equals

$$
\begin{aligned}
\sum_{m} & (-1)^{m} q_{1}^{m^{2}+m} \alpha^{2 m}\left(i \sqrt{\frac{5-\sqrt{5}}{2}} q_{1}^{-1} \psi\left(-q_{1}^{5}\right)+i \sqrt{\frac{5+\sqrt{5}}{2}} q_{1} \phi\left(-q_{1}^{5}\right)\right) \\
+ & \left(1+\alpha^{8}\right)\left(-q_{1} a_{1}\left(-q_{1}^{5}\right) f\left(-q_{1}^{10},-q_{1}^{40}\right)+2 q_{1}^{6} A\left(q_{1}^{30},-q_{1}^{5},-q_{1}^{25}\right)\right. \\
& -2 q_{1}^{12} A\left(q_{1}^{-20}, q_{1}^{10},-q_{1}^{25}\right)+q_{1}^{-1} a_{1}\left(-q_{1}^{5}\right) f\left(-q_{1}^{20},-q_{1}^{30}\right)+q_{1}^{3} a_{2}\left(-q_{1}^{5}\right) f\left(-q_{1}^{10},-q_{1}^{40}\right) \\
& \left.-2 q_{1}^{8} A\left(q_{1}^{20}, q_{1}^{10},-q_{1}^{25}\right)+\frac{f\left(-q_{1}^{5}, q_{1}^{20}\right)\left(-q_{1}^{25} ;-q_{1}^{25}\right)_{\infty}\left(q_{1}^{50} ; q_{1}^{50}\right)_{\infty}}{f\left(q_{1}^{5}, q_{1}^{45}\right)}\right) \\
+ & \left(\alpha^{2}+\alpha^{6}\right)\left(2 q_{1}^{10} A\left(q_{1}^{-10},-q_{1}^{5},-q_{1}^{25}\right)-q_{1}^{3} a_{2}\left(-q_{1}^{5}\right) f\left(-q_{1}^{10},-q_{1}^{40}\right)\right. \\
& -q_{1}^{-1} a_{1}\left(-q_{1}^{5}\right) f\left(-q_{1}^{20},-q_{1}^{30}\right)+2 q_{1}^{4} A\left(q_{1}^{10},-q_{1}^{5},-q_{1}^{25}\right)-q_{1} a_{2}\left(-q_{1}^{5}\right) f\left(-q_{1}^{20},-q_{1}^{30}\right) \\
& \left.+2 q_{1}^{6} A\left(q_{1}^{10}, q_{1}^{10},-q_{1}^{25}\right)-q^{2} \frac{f\left(-q_{1}^{15}, q_{1}^{10}\right)\left(-q_{1}^{25} ;-q_{1}^{25}\right)_{\infty}\left(q_{1}^{50} ; q_{1}^{50}\right)_{\infty}}{f\left(q_{1}^{15}, q_{1}^{35}\right)}\right) \\
+ & 2 \alpha^{4} q_{1}\left(a_{1}\left(-q_{1}^{5}\right) f\left(-q_{1}^{10},-q_{1}^{40}\right)+a_{2}\left(-q_{1}^{5}\right) f\left(-q_{1}^{20},-q_{1}^{30}\right)-2 q_{1}^{5} f\left(-q_{1}^{25},-q_{1}^{75}\right)^{2}\right. \\
& \left.-q_{1}^{5} f\left(-q_{1}^{25},-q_{1}^{75}\right)^{2}\right) \\
= & \sum_{m}(-1)^{m} q_{1}^{m^{2}+m} \alpha^{2 m}\left(i \sqrt{\frac{5-\sqrt{5}}{2}} q_{1}^{-1} \psi\left(-q_{1}^{5}\right)+i \sqrt{\frac{5+\sqrt{5}}{2}} q_{1} \phi\left(-q_{1}^{5}\right)\right) \\
& +\left(1+\alpha^{8}\right) \frac{f\left(-q_{1},-q_{1}^{9}\right)^{2} f\left(-q_{1}^{8},-q_{1}^{12}\right)\left(q_{1}^{10} ; q_{1}^{10}\right)_{\infty}\left(q_{1}^{20} ; q_{1}^{20}\right)_{\infty}}{f\left(-q_{1}^{3},-q_{1}^{7}\right) f\left(-q_{1}^{2},-q_{1}^{18}\right) f\left(-q_{1}^{4},-q_{1}^{16}\right)} \\
& -\left(\alpha^{2}+\alpha^{6}\right) q_{1} \frac{f\left(-q_{1}^{3},-q_{1}^{7}\right)^{2} f\left(-q_{1}^{4},-q_{1}^{16}\right)\left(q_{1}^{10} ; q_{1}^{10}\right)_{\infty}\left(q_{1}^{20} ; q_{1}^{20}\right)_{\infty}}{f\left(-q_{1},-q_{1}^{9}\right) f\left(-q_{1}^{6},-q_{1}^{14}\right) f\left(-q_{1}^{8},-q_{1}^{12}\right)} \\
& +2 \alpha^{4} q_{1} f\left(-q_{1}^{5},-q_{1}^{15}\right)^{2} .
\end{aligned}
$$

Therefore, by (3.9), (3.16), and (3.18), we derive

$$
\begin{aligned}
& -\frac{\sqrt{5+\sqrt{5}}}{5 n \sqrt{2}}\left(e^{-\frac{\pi n}{5}+\pi n} \frac{F\left(\frac{2}{5}, \frac{i}{5 n}\right)}{\theta_{11}(2 i n, 5 i n)}-e^{-\frac{\pi n}{5}+2 \pi n} \frac{F\left(\frac{3}{5}, \frac{i}{5 n}\right)}{\theta_{11}(3 i n, 5 i n)}\right) \\
& =-\frac{1}{\sqrt{n}} q_{1}^{-1} \psi\left(-q_{1}^{5}\right)-\frac{\sqrt{5}+1}{2 \sqrt{n}} q_{1} \phi\left(-q_{1}^{5}\right)+i \frac{\sqrt{5+\sqrt{5}}}{\sqrt{10 n}} \frac{1}{\sum_{m}(-1)^{m} q_{1}^{m^{2}+m} \alpha^{2 m}} \\
& \times\left(\left(1+\alpha^{8}\right) \frac{f\left(-q_{1},-q_{1}^{9}\right)^{2} f\left(-q_{1}^{8},-q_{1}^{12}\right)\left(q_{1}^{10} ; q_{1}^{10}\right)_{\infty}\left(q_{1}^{20} ; q_{1}^{20}\right)_{\infty}}{f\left(-q_{1}^{3},-q_{1}^{7}\right) f\left(-q_{1}^{2},-q_{1}^{18}\right) f\left(-q_{1}^{4},-q_{1}^{16}\right)}\right. \\
& \quad-\left(\alpha^{2}+\alpha^{6}\right) q_{1} \frac{f\left(-q_{1}^{3},-q_{1}^{7}\right)^{2} f\left(-q_{1}^{4},-q_{1}^{16}\right)\left(q_{1}^{10} ; q_{1}^{10}\right)_{\infty}\left(q_{1}^{20} ; q_{1}^{20}\right)_{\infty}}{f\left(-q_{1},-q_{1}^{9}\right) f\left(-q_{1}^{6},-q_{1}^{14}\right) f\left(-q_{1}^{8},-q_{1}^{12}\right)} \\
& \left.\quad+2 \alpha^{4} q_{1} f\left(-q_{1}^{5},-q_{1}^{15}\right)^{2}\right) .
\end{aligned}
$$


To complete the proof of Theorem 3.1, we need the following identities. Using (1.3), we can derive that $\sum_{m}(-1)^{m} q_{1}^{m^{2}+m} \gamma^{2 m}$ equals

$$
\left\{\begin{array}{c}
(1-\gamma) f\left(-q_{1}^{6},-q_{1}^{12}\right) \\
\text { if } \gamma=e^{\pi i / 3} \\
\left(1-\gamma^{8}\right) f\left(-q_{1}^{20},-q_{1}^{30}\right)-\left(\gamma^{2}-\gamma^{6}\right) q_{1}^{2} f\left(-q_{1}^{10},-q_{1}^{40}\right) \\
\text { if } \gamma=e^{\pi i / 5} \\
\left(1+\gamma^{8}\right) f\left(q_{1}^{20}, q_{1}^{30}\right)-\left(\gamma^{2}+\gamma^{6}\right) q_{1}^{2} f\left(q_{1}^{10}, q_{1}^{40}\right)+2 \gamma^{4} q_{1}^{6} f\left(q_{1}^{50}, q_{1}^{150}\right) \\
\text { if } \gamma=e^{\pi i / 10} \text { or } \gamma=e^{\pi 3 i / 10}
\end{array}\right.
$$

and $\sum_{m}(-1)^{m} q_{1}^{m^{2}} \gamma^{2 m}$ equals

$$
\left\{\begin{array}{c}
f\left(-q_{1}^{25},-q_{1}^{25}\right)-\left(\gamma^{2}+\gamma^{8}\right) q_{1} f\left(-q_{1}^{15},-q_{1}^{35}\right)+\left(\gamma^{4}+\gamma^{6}\right) q_{1}^{4} f\left(-q_{1}^{5},-q_{1}^{45}\right) \\
\text { if } \gamma=e^{\pi i / 5} \text { or } e^{2 \pi i / 5} \\
f\left(q_{1}^{25}, q_{1}^{25}\right)-\left(\gamma^{2}-\gamma^{8}\right) q_{1} f\left(q_{1}^{15}, q_{1}^{35}\right)+\left(\gamma^{4}-\gamma^{6}\right) q_{1}^{4} f\left(q_{1}^{5}, q_{1}^{45}\right) \\
\text { if } \gamma=e^{\pi i / 10} \text { or } e^{3 \pi i / 10}
\end{array}\right.
$$

Recall that $q=e^{-\pi n}$ and $q_{1}=e^{-\frac{\pi}{5 n}}$. By (1.3), (1.16), and (3.6), we are able to derive the following four identities:

$$
\left\{\begin{array}{l}
f\left(-q,-q^{9}\right)=-\frac{i}{\sqrt{5 n}} e^{\frac{4 n \pi}{5}-\frac{\pi}{20 n}+\frac{\pi i}{10}} \sum_{m}(-1)^{m} q_{1}^{m^{2}+m} e^{2 m \frac{\pi i}{10}} \\
f\left(q, q^{9}\right)=\frac{1}{\sqrt{5 n}} e^{\frac{4 n \pi}{5}} \sum_{m}(-1)^{m} q_{1}^{m^{2}} e^{2 m \frac{\pi i}{10}} \\
f\left(q^{3}, q^{7}\right)=\frac{1}{\sqrt{5 n}} e^{\frac{n \pi}{5}} \sum_{m}(-1)^{m} q_{1}^{m^{2}} e^{2 m \frac{3 \pi i}{10}} \\
f\left(q^{4}, q^{6}\right)=\frac{1}{\sqrt{5 n}} e^{\frac{n \pi}{20}} \sum_{m}(-1)^{m} q_{1}^{m^{2}} e^{2 m \frac{2 \pi i}{5}}
\end{array}\right.
$$

By (3.22), we find that

$$
\begin{aligned}
& \frac{f\left(-q,-q^{9}\right) f\left(q^{3}, q^{7}\right) f\left(q^{4}, q^{6}\right)}{f\left(q, q^{9}\right)}=\frac{1}{5 n} e^{-\frac{\pi}{20 n}+\frac{n \pi}{5}+\frac{n \pi}{20}} \\
& \quad \times \frac{\alpha^{4} \sum_{m}(-1)^{m} q_{1}^{m^{2}+m} e^{2 m \frac{\pi i}{10}} \sum_{m}(-1)^{m} q_{1}^{m^{2}} e^{2 m \frac{3 \pi i}{10}} \sum_{m}(-1)^{m} q_{1}^{m^{2}} e^{2 m \frac{2 \pi i}{5}}}{\sum_{m}(-1)^{m} q_{1}^{m^{2}} e^{2 m \frac{\pi i}{10}}} .
\end{aligned}
$$

Recall that $\alpha=e^{2 \pi i / 5}$ and $\alpha^{5}=1$. By (3.20), (3,21), Theorem 2.22 with $q$ replaced by $q_{1}$, and Theorem 2.6 with $q$ replaced by $q_{1}$, the numerator of the right side of (3.23) equals

$$
\begin{aligned}
\alpha^{4} & \left(\left(1+\alpha^{2}\right) f\left(q_{1}^{20}, q_{1}^{30}\right)+\left(\alpha^{3}+\alpha^{4}\right) q_{1}^{2} f\left(q_{1}^{10}, q_{1}^{40}\right)+2 \alpha q_{1}^{6} f\left(q_{1}^{50}, q_{1}^{150}\right)\right) \\
& \times\left(f\left(q_{1}^{25}, q_{1}^{25}\right)+\left(\alpha+\alpha^{4}\right) q_{1} f\left(q_{1}^{15}, q_{1}^{35}\right)+\left(\alpha^{2}+\alpha^{3}\right) q_{1}^{4} f\left(q_{1}^{5}, q_{1}^{45}\right)\right) \\
& \times\left(f\left(-q_{1}^{25},-q_{1}^{25}\right)-\left(\alpha^{2}+\alpha^{3}\right) q_{1} f\left(-q_{1}^{15},-q_{1}^{35}\right)+\left(\alpha+\alpha^{4}\right) q_{1}^{4} f\left(-q_{1}^{5},-q_{1}^{45}\right)\right) \\
= & \alpha^{4}\left(\left(1+\alpha^{2}\right) f\left(q_{1}^{20}, q_{1}^{30}\right)+\left(\alpha^{3}+\alpha^{4}\right) q_{1}^{2} f\left(q_{1}^{10}, q_{1}^{40}\right)+2 \alpha q_{1}^{6} f\left(q_{1}^{50}, q_{1}^{150}\right)\right) \\
& \times\left(\frac{f\left(-q_{1},-q_{1}^{9}\right)\left(q_{1}^{4} ; q_{1}^{4}\right)_{\infty}\left(q_{1}^{10} ; q_{1}^{10}\right)_{\infty}^{2}}{f\left(-q_{1}^{3},-q_{1}^{7}\right) f\left(-q_{1}^{2},-q_{1}^{18}\right)}-2\left(\alpha^{2}+\alpha^{3}\right) q_{1} f\left(-q_{1}^{4},-q_{1}^{16}\right) f\left(-q_{1}^{8},-q_{1}^{12}\right)\right)
\end{aligned}
$$




$$
\begin{aligned}
= & \left(\alpha+\alpha^{4}\right) f\left(q_{1}^{20}, q_{1}^{30}\right) \frac{f\left(-q_{1},-q_{1}^{9}\right)\left(q_{1}^{4} ; q_{1}^{4}\right)_{\infty}\left(q_{1}^{10} ; q_{1}^{10}\right)_{\infty}^{2}}{f\left(-q_{1}^{3},-q_{1}^{7}\right) f\left(-q_{1}^{2},-q_{1}^{18}\right)} \\
& +2 q_{1} f\left(q_{1}^{20}, q_{1}^{30}\right) f\left(-q_{1}^{4},-q_{1}^{16}\right) f\left(-q_{1}^{8},-q_{1}^{12}\right) \\
& +\left(\alpha^{2}+\alpha^{3}\right) q_{1}^{2} f\left(q_{1}^{10}, q_{1}^{40}\right) \frac{f\left(-q_{1},-q_{1}^{9}\right)\left(q_{1}^{4} ; q_{1}^{4}\right)_{\infty}\left(q_{1}^{10} ; q_{1}^{10}\right)_{\infty}^{2}}{f\left(-q_{1}^{3},-q_{1}^{7}\right) f\left(-q_{1}^{2},-q_{1}^{18}\right)} \\
& -2\left(2+\alpha+\alpha^{4}\right) q_{1}^{3} f\left(q_{1}^{10}, q_{1}^{40}\right) f\left(-q_{1}^{4},-q_{1}^{16}\right) f\left(-q_{1}^{8},-q_{1}^{12}\right) \\
& +2 q_{1}^{6} f\left(q_{1}^{50}, q_{1}^{150}\right) \frac{f\left(-q_{1},-q_{1}^{9}\right)\left(q_{1}^{4} ; q_{1}^{4}\right)_{\infty}\left(q_{1}^{10} ; q_{1}^{10}\right)_{\infty}^{2}}{f\left(-q_{1}^{3},-q_{1}^{7}\right) f\left(-q_{1}^{2},-q_{1}^{18}\right)} \\
& -4\left(\alpha^{2}+\alpha^{3}\right) q_{1}^{7} f\left(q_{1}^{50}, q_{1}^{150}\right) f\left(-q_{1}^{4},-q_{1}^{16}\right) f\left(-q_{1}^{8},-q_{1}^{12}\right) .
\end{aligned}
$$

Here, we easily calculate that $\alpha+\alpha^{4}=\frac{-1+\sqrt{5}}{2}, \alpha^{2}+\alpha^{3}=\frac{-1-\sqrt{5}}{2}$, and $2+\alpha+$ $\alpha^{4}=\frac{3+\sqrt{5}}{2}$. Now, let $L S_{1}=-\frac{1}{2} \times$ the left side of (2.31) with $q$ replaced by $q_{1}$, $L S_{2}=\frac{\sqrt{5}}{2} \times$ the left side of $(2.32)$ with $q$ replaced by $q_{1}, R S_{1}=-\frac{1}{2} \times$ the right side of (2.31) with $q$ replaced by $q_{1}$, and $R S_{2}=\frac{\sqrt{5}}{2} \times$ the right side of $(2.32)$ with $q$ replaced by $q_{1}$. Then, we easily verify that the right side of (3.24) equals the sum of $L S_{1}$ and $L S_{2}$. Therefore, we can say that the right side of (3.24) equals the sum of $R S_{1}$ and $R S_{2}$. Using (3.21), we can find that the sum of $R S_{1}$ and $R S_{2}$ equals

$$
\begin{aligned}
& \left(\alpha+\alpha^{4}\right) f\left(q_{1}^{25}, q_{1}^{25}\right) \frac{f\left(-q_{1},-q_{1}^{9}\right)^{2} f\left(-q_{1}^{8},-q_{1}^{12}\right)\left(q_{1}^{10} ; q_{1}^{10}\right)_{\infty}\left(q_{1}^{20} ; q_{1}^{20}\right)_{\infty}}{f\left(-q_{1}^{3},-q_{1}^{7}\right) f\left(-q_{1}^{2},-q_{1}^{18}\right) f\left(-q_{1}^{4},-q_{1}^{16}\right)} \\
& +2 q_{1} f\left(q_{1}^{25}, q_{1}^{25}\right) f\left(-q_{1}^{5},-q_{1}^{15}\right)^{2} \\
& -\left(\alpha^{2}+\alpha^{3}\right) q_{1} f\left(q_{1}^{25}, q_{1}^{25}\right) \frac{f\left(-q_{1}^{3},-q_{1}^{7}\right)^{2} f\left(-q_{1}^{4},-q_{1}^{16}\right)\left(q_{1}^{10} ; q_{1}^{10}\right)_{\infty}\left(q_{1}^{20} ; q_{1}^{20}\right)_{\infty}}{f\left(-q_{1},-q_{1}^{9}\right) f\left(-q_{1}^{6},-q_{1}^{14}\right) f\left(-q_{1}^{8},-q_{1}^{12}\right)} \\
& -q_{1} f\left(q_{1}^{15}, q_{1}^{35}\right) \frac{f\left(-q_{1},-q_{1}^{9}\right)^{2} f\left(-q_{1}^{8},-q_{1}^{12}\right)\left(q_{1}^{10} ; q_{1}^{10}\right)_{\infty}\left(q_{1}^{20} ; q_{1}^{20}\right)_{\infty}}{f\left(-q_{1}^{3},-q_{1}^{7}\right) f\left(-q_{1}^{2},-q_{1}^{18}\right) f\left(-q_{1}^{4},-q_{1}^{16}\right)} \\
& +2\left(\alpha^{2}+\alpha^{3}\right) q_{1}^{2} f\left(q_{1}^{15}, q_{1}^{35}\right) f\left(-q_{1}^{5},-q_{1}^{15}\right)^{2} \\
& -\left(2+\alpha+\alpha^{4}\right) q_{1}^{2} f\left(q_{1}^{15}, q_{1}^{35}\right) \frac{f\left(-q_{1}^{3},-q_{1}^{7}\right)^{2} f\left(-q_{1}^{4},-q_{1}^{16}\right)\left(q_{1}^{10} ; q_{1}^{10}\right)_{\infty}\left(q_{1}^{20} ; q_{1}^{20}\right)_{\infty}}{f\left(-q_{1},-q_{1}^{9}\right) f\left(-q_{1}^{6},-q_{1}^{14}\right) f\left(-q_{1}^{8},-q_{1}^{12}\right)} \\
& +\left(2+\alpha^{2}+\alpha^{3}\right) q_{1}^{4} f\left(q_{1}^{5}, q_{1}^{45}\right) \frac{f\left(-q_{1},-q_{1}^{9}\right)^{2} f\left(-q_{1}^{8},-q_{1}^{12}\right)\left(q_{1}^{10} ; q_{1}^{10}\right)_{\infty}\left(q_{1}^{20} ; q_{1}^{20}\right)_{\infty}}{f\left(-q_{1}^{3},-q_{1}^{7}\right) f\left(-q_{1}^{2},-q_{1}^{18}\right) f\left(-q_{1}^{4},-q_{1}^{16}\right)} \\
& +2\left(\alpha+\alpha^{4}\right) q_{1}^{5} f\left(q_{1}^{5}, q_{1}^{45}\right) f\left(-q_{1}^{5},-q_{1}^{15}\right)^{2} \\
& +q_{1}^{5} f\left(q_{1}^{5}, q_{1}^{45}\right) \frac{f\left(-q_{1}^{3},-q_{1}^{7}\right)^{2} f\left(-q_{1}^{4},-q_{1}^{16}\right)\left(q_{1}^{10} ; q_{1}^{10}\right)_{\infty}\left(q_{1}^{20} ; q_{1}^{20}\right)_{\infty}}{f\left(-q_{1},-q_{1}^{9}\right) f\left(-q_{1}^{6},-q_{1}^{14}\right) f\left(-q_{1}^{8},-q_{1}^{12}\right)} \\
& =\alpha\left(f\left(q_{1}^{25}, q_{1}^{25}\right)+\left(\alpha^{2}+\alpha^{3}\right) q_{1} f\left(q_{1}^{15}, q_{1}^{35}\right)+\left(\alpha+\alpha^{4}\right) q_{1}^{4} f\left(q_{1}^{5}, q_{1}^{45}\right)\right) \\
& \times\left(\left(1+\alpha^{8}\right) \frac{f\left(-q_{1},-q_{1}^{9}\right)^{2} f\left(-q_{1}^{8},-q_{1}^{12}\right)\left(q_{1}^{10} ; q_{1}^{10}\right)_{\infty}\left(q_{1}^{20} ; q_{1}^{20}\right)_{\infty}}{f\left(-q_{1}^{3},-q_{1}^{7}\right) f\left(-q_{1}^{2},-q_{1}^{18}\right) f\left(-q_{1}^{4},-q_{1}^{16}\right)}\right. \\
& -\left(\alpha^{2}+\alpha^{6}\right) q_{1} \frac{f\left(-q_{1}^{3},-q_{1}^{7}\right)^{2} f\left(-q_{1}^{4},-q_{1}^{16}\right)\left(q_{1}^{10} ; q_{1}^{10}\right)_{\infty}\left(q_{1}^{20} ; q_{1}^{20}\right)_{\infty}}{f\left(-q_{1},-q_{1}^{9}\right) f\left(-q_{1}^{6},-q_{1}^{14}\right) f\left(-q_{1}^{8},-q_{1}^{12}\right)} \\
& \left.+2 \alpha^{4} q_{1} f\left(-q_{1}^{5},-q_{1}^{15}\right)^{2}\right)
\end{aligned}
$$




$$
\begin{aligned}
=\alpha \sum_{m} & (-1)^{m} q_{1}^{m^{2}} e^{2 m \frac{\pi i}{10}} \\
& \times\left(\left(1+\alpha^{8}\right) \frac{f\left(-q_{1},-q_{1}^{9}\right)^{2} f\left(-q_{1}^{8},-q_{1}^{12}\right)\left(q_{1}^{10} ; q_{1}^{10}\right)_{\infty}\left(q_{1}^{20} ; q_{1}^{20}\right)_{\infty}}{f\left(-q_{1}^{3},-q_{1}^{7}\right) f\left(-q_{1}^{2},-q_{1}^{18}\right) f\left(-q_{1}^{4},-q_{1}^{16}\right)}\right. \\
& \quad-\left(\alpha^{2}+\alpha^{6}\right) q_{1} \frac{f\left(-q_{1}^{3},-q_{1}^{7}\right)^{2} f\left(-q_{1}^{4},-q_{1}^{16}\right)\left(q_{1}^{10} ; q_{1}^{10}\right)_{\infty}\left(q_{1}^{20} ; q_{1}^{20}\right)_{\infty}}{f\left(-q_{1},-q_{1}^{9}\right) f\left(-q_{1}^{6},-q_{1}^{14}\right) f\left(-q_{1}^{8},-q_{1}^{12}\right)} \\
& \left.\quad+2 \alpha^{4} q_{1} f\left(-q_{1}^{5},-q_{1}^{15}\right)^{2}\right) .
\end{aligned}
$$

By (3.7), (1.3), and (1.16), we find that

$$
f\left(-q^{4},-q^{6}\right)=\frac{\sum_{m}(-1)^{m} q_{1}^{m^{2}+m} \alpha^{2 m}}{i \sqrt{5 n} \alpha^{-1} e^{\frac{\pi}{20 n}} e^{-\frac{n \pi}{20}}} .
$$

By (3.23), (3.24), (3.25), and (3.26), we find that

$$
\begin{aligned}
& -\sqrt{\frac{5+\sqrt{5}}{2}} e^{-\frac{\pi n}{5}} \frac{f\left(-q,-q^{9}\right) f\left(q^{3}, q^{7}\right) f\left(q^{4}, q^{6}\right)}{f\left(-q^{4},-q^{6}\right) f\left(q, q^{9}\right)} \\
& =-\sqrt{\frac{5+\sqrt{5}}{2}} \frac{1}{5 n} e^{-\frac{\pi}{20 n}+\frac{n \pi}{20}} i \sqrt{5 n} \alpha^{-1} e^{\frac{\pi}{20 n}} e^{-\frac{n \pi}{20}} \\
& \times \frac{\alpha^{4} \sum_{m}(-1)^{m} q_{1}^{m^{2}+m} e^{2 m \frac{\pi i}{10}} \sum_{m}(-1)^{m} q_{1}^{m^{2}} e^{2 m \frac{3 \pi i}{10}} \sum_{m}(-1)^{m} q_{1}^{m^{2}} e^{2 m \frac{2 \pi i}{5}}}{\sum_{m}(-1)^{m} q_{1}^{\left(m^{2}+m\right)} \alpha^{2 m} \sum_{m}(-1)^{m} q_{1}^{m^{2}} e^{2 m \frac{\pi i}{10}}} \\
& =-\sqrt{\frac{5+\sqrt{5}}{10 n}} \frac{i}{\sum_{m}(-1)^{m} q_{1}^{m^{2}+m} \alpha^{2 m}} \\
& \quad \times\left(\left(1+\alpha^{8}\right) \frac{f\left(-q_{1},-q_{1}^{9}\right)^{2} f\left(-q_{1}^{8},-q_{1}^{12}\right)\left(q_{1}^{10} ; q_{1}^{10}\right)_{\infty}\left(q_{1}^{20} ; q_{1}^{20}\right)_{\infty}}{f\left(-q_{1}^{3},-q_{1}^{7}\right) f\left(-q_{1}^{2},-q_{1}^{18}\right) f\left(-q_{1}^{4},-q_{1}^{16}\right)}\right. \\
& \quad-\left(\alpha^{2}+\alpha^{6}\right) q_{1} \frac{f\left(-q_{1}^{3},-q_{1}^{7}\right)^{2} f\left(-q_{1}^{4},-q_{1}^{16}\right)\left(q_{1}^{10} ; q_{1}^{10}\right)_{\infty}\left(q_{1}^{20} ; q_{1}^{20}\right)_{\infty}}{f\left(-q_{1},-q_{1}^{9}\right) f\left(-q_{1}^{6},-q_{1}^{14}\right) f\left(-q_{1}^{8},-q_{1}^{12}\right)} \\
& \left.\quad+2 \alpha^{4} q_{1} f\left(-q_{1}^{5},-q_{1}^{15}\right)^{2}\right) .
\end{aligned}
$$

In conclusion, using (3.4), (3.5), (3.19), and (3.27), we see that we have completed the proof of Ramanujan's seventh identity.

\section{Proof of Ramanujan's eighth identity}

In this section, we will prove the Ramanujan's eighth identity (1.2). The proof of eighth identity is similar to that of seventh identity.

Theorem 4.1 (Ramanujan's eighth tenth order mock theta function identity).

$$
\begin{gathered}
\int_{0}^{\infty} \frac{e^{-\pi n x^{2}}}{\cosh \frac{2 \pi x}{\sqrt{5}}+\frac{1-\sqrt{5}}{4}} d x+\frac{1}{\sqrt{n}} e^{\frac{\pi}{5 n}} \psi\left(-e^{-\frac{\pi}{n}}\right) \\
=-\sqrt{\frac{5-\sqrt{5}}{2}} e^{\frac{\pi n}{5}} \psi\left(-e^{-\pi n}\right)+\frac{\sqrt{5}-1}{2 \sqrt{n}} e^{-\frac{\pi}{5 n}} \phi\left(-e^{-\frac{\pi}{n}}\right) .
\end{gathered}
$$


Proof. Replacing $\theta^{\prime}$ by $\frac{1}{5}$ in (3.1), and using (1.14), we find that

$$
\int_{-\infty}^{\infty} \frac{e^{-\pi n z^{2}}}{e^{\frac{2 \pi z}{\sqrt{5}}}+e^{-\frac{3 \pi i}{5}}} d z=\sqrt{5} e^{\frac{\pi n}{5}-\frac{2 \pi i}{5}} \frac{F\left(\frac{1}{5}, \frac{i}{5 n}\right)-5 n F(i n, 5 i n)}{5 i n \theta_{11}(i n, 5 i n)} .
$$

Similarly, replacing $\theta^{\prime}$ by $\frac{4}{5}$ in (3.1), and using (1.14), we find that

$$
\int_{-\infty}^{\infty} \frac{e^{-\pi n z^{2}}}{e^{\frac{2 \pi z}{\sqrt{5}}}+e^{\frac{3 \pi i}{5}}} d z=\sqrt{5} e^{\frac{16 \pi n}{5}-\frac{8 \pi i}{5}} \frac{F\left(\frac{4}{5}, \frac{i}{5 n}\right)-5 n F(4 i n, 5 i n)}{5 i n \theta_{11}(4 i n, 5 i n)} .
$$

Using (4.1) and (4.2), we find that

$$
\begin{aligned}
& \int_{0}^{\infty} \frac{e^{-\pi n x^{2}}}{\cosh \frac{2 \pi x}{\sqrt{5}}+\frac{1-\sqrt{5}}{4}} d x=\frac{1}{2} \int_{-\infty}^{\infty} \frac{e^{-\pi n x^{2}}}{\cosh \frac{2 \pi x}{\sqrt{5}}+\frac{1-\sqrt{5}}{4}} d x \\
& =-\frac{1}{2} \int_{-\infty}^{\infty} \frac{e^{-\pi n x^{2}}}{i \sin \frac{3 \pi}{5}}\left(\frac{1}{1+e^{\frac{2 \pi x}{\sqrt{5}}+\frac{3 \pi}{5} i}}-\frac{1}{1+e^{\frac{2 \pi x}{\sqrt{5}}-\frac{3 \pi}{5} i}}\right) d x \\
& =-\frac{1}{2 i \sin \frac{3 \pi}{5}}\left(e^{-\frac{3 \pi}{5} i} \int_{-\infty}^{\infty} \frac{e^{-\pi n x^{2}}}{e^{\frac{2 \pi x}{\sqrt{5}}}+e^{-\frac{3 \pi}{5} i}} d x-e^{\frac{3 \pi}{5} i} \int_{-\infty}^{\infty} \frac{e^{-\pi n x^{2}}}{e^{\frac{2 \pi x}{\sqrt{5}}}+e^{\frac{3 \pi}{5} i}} d x\right) \\
& (4.3) \\
& =-\frac{\sqrt{5-\sqrt{5}}}{5 n \sqrt{2}}\left(e^{\frac{\pi n}{5}} \frac{F\left(\frac{1}{5}, \frac{i}{5 n}\right)-5 n F(i n, 5 i n)}{\theta_{11}(i n, 5 i n)}-e^{\frac{16 \pi n}{5}} \frac{F\left(\frac{4}{5}, \frac{i}{5 n}\right)-5 n F(4 i n, 5 i n)}{\theta_{11}(4 i n, 5 i n)}\right) .
\end{aligned}
$$

Let $q=e^{-\pi n}$. Then, using (1.15), (1.16), (1.3), (1.9) with $q$ and $z$ replaced by $-q$ and $q^{2}$, respectively, (1.7), (1.11) with $q, z$, and $x$ replaced by $-q^{5}, 1$ and $-q$, respectively, (1.12) with $q$ replaced by $-q$, and Theorem 2.5 , we can derive that

$$
\begin{aligned}
& \sqrt{\frac{5-\sqrt{5}}{2}}\left(e^{\frac{\pi n}{5}} \frac{F(\text { in }, 5 i n)}{\theta_{11}(i n, 5 i n)}-e^{\frac{\pi n}{5}+3 \pi n} \frac{F(4 i n, 5 i n)}{\theta_{11}(4 i n, 5 i n)}\right) \\
& =-\sqrt{\frac{5-\sqrt{5}}{2}} e^{\frac{\pi n}{5}}\left(\frac{\sum_{m} \frac{(-1)^{m} q^{5 m^{2}+7 m+2}}{1+q^{10 m+5}}}{f\left(-q^{2},-q^{8}\right)}+\frac{\sum_{m} \frac{(-1)^{m} q^{5 m^{2}+7 m+2}}{1+q^{10 m+5}}}{f\left(-q^{2},-q^{8}\right)}\right) \\
& =-\sqrt{\frac{5-\sqrt{5}}{2}} e^{\frac{\pi n}{5}} \frac{L_{1}\left(q, q^{2}\right)}{f\left(-q^{2},-q^{8}\right)} \\
& =-\sqrt{\frac{5-\sqrt{5}}{2}} e^{\frac{\pi n}{5}}\left(\psi(-q)-q \frac{\left(-q^{5} ;-q^{5}\right)_{\infty}\left(q^{10} ; q^{10}\right)_{\infty} f\left(-q^{2},-q^{8}\right)}{f\left(q,-q^{4}\right) f\left(-q^{4},-q^{6}\right)}\right. \\
& \left.+2 q \frac{\left(-q^{5} ;-q^{5}\right)_{\infty}^{2}\left(q^{10} ; q^{10}\right)_{\infty}^{2}}{f\left(q^{5}, q^{5}\right)^{2} f\left(-q^{2},-q^{8}\right)}\right) \\
& =-\sqrt{\frac{5-\sqrt{5}}{2}} e^{\frac{\pi n}{5}} \psi(-q)-\sqrt{\frac{5-\sqrt{5}}{2}} e^{\frac{\pi n}{5}} q \frac{f\left(-q^{3},-q^{7}\right) f\left(q, q^{9}\right) f\left(q^{2}, q^{8}\right)}{f\left(-q^{2},-q^{8}\right) f\left(q^{3}, q^{7}\right)} .
\end{aligned}
$$

Replacing $x$ and $\omega$ by in and 5in, respectively, in (3.6), we find that

$$
\theta_{11}\left(\frac{1}{5}, \frac{i}{5 n}\right)=-i \sqrt{5 n} e^{-\frac{n \pi}{5}} \theta_{11}(i n, 5 i n) .
$$


Replacing $x$ and $\omega$ by $4 i n$ and $5 i n$, respectively, in (3.6), we find that

$$
\theta_{11}\left(\frac{4}{5}, \frac{i}{5 n}\right)=-i \sqrt{5 n} e^{-\frac{16 n \pi}{5}} \theta_{11}(4 i n, 5 i n) .
$$

Let $q_{1}=e^{-\frac{\pi}{5 n}}, \alpha^{\prime}=e^{\frac{\pi i}{5}}$, and $\beta^{\prime}=e^{\frac{4 \pi i}{5}}=-\alpha^{\prime-1}$. Then, using (4.5), (4.6), (1.15), and (1.16), we find that

$$
\begin{aligned}
\frac{i}{\sqrt{5 n}}\left(e^{\frac{n \pi}{5}} \frac{F\left(\frac{1}{5}, \frac{i}{5 n}\right)}{\theta_{11}(i n, 5 i n)}-e^{\frac{16 n \pi}{5}}\right. & \left.\frac{F\left(\frac{4}{5}, \frac{i}{5 n}\right)}{\theta_{11}(4 i n, 5 i n)}\right)=\frac{F\left(\frac{1}{5}, \frac{i}{5 n}\right)}{\theta_{11}\left(\frac{1}{5}, \frac{i}{5 n}\right)}-\frac{F\left(\frac{4}{5}, \frac{i}{5 n}\right)}{\theta_{11}\left(\frac{4}{5}, \frac{i}{5 n}\right)} \\
= & \frac{\sum_{m} \frac{(-1)^{m} q_{1}^{m^{2}+m} \alpha^{2 m}\left(1-q_{1}^{2 m+1}\right)}{1+q_{1}^{2 m+1}}}{\sum_{m}(-1)^{m} q_{1}^{m^{2}+m} \alpha^{2 m}} .
\end{aligned}
$$

If we replace $\alpha$ by $\alpha^{\prime}$ in the right side of (3.9), we can find that the right side of (3.9) equals the right side of (4.7). And, it's easy to verify that (3.10), (3.11), and (3.16) are correct if we replace $\alpha$ by $\alpha^{\prime}$ in (3.10), (3.11), and (3.16). Therefore, we find that

$$
\sum_{m}(-1)^{m} q_{1}^{m^{2}+m} \alpha^{\prime 2 m}=\left(1-\alpha^{\prime 8}\right) f\left(-q_{1}^{20},-q_{1}^{30}\right)-q_{1}^{2}\left(\alpha^{2}-\alpha^{6}\right) f\left(-q_{1}^{10},-q_{1}^{40}\right),
$$

and the numerator of (4.7) equals

$$
\begin{aligned}
& \left(1+\alpha^{\prime 8}\right)\left(q_{1} L_{1}\left(q_{1}^{5}, q_{1}^{40}\right)-q_{1}^{7} L_{2}\left(q_{1}^{5}, 1\right)-q^{-1} L_{1}\left(q^{5}, q^{30}\right)-q^{3} L_{2}\left(q^{5}, q^{40}\right)\right) \\
& +\left(\alpha^{2}+\alpha^{\prime 6}\right)\left(q^{5} L_{1}\left(q^{5}, 1\right)+q^{3} L_{2}\left(q^{5}, q^{10}\right)+q^{-1} L_{1}\left(q^{5}, q^{20}\right)+q L_{2}\left(q^{5}, q^{30}\right)\right) \\
& -2 \alpha^{\prime 4}\left(q L_{1}\left(q^{5}, q^{10}\right)+q L_{2}\left(q^{5}, q^{20}\right)\right) \\
& +\left(1+\alpha^{\prime 8}\right) \frac{f\left(-q_{1}^{5}, q_{1}^{20}\right)\left(-q_{1}^{25} ;-q_{1}^{25}\right)_{\infty}\left(q_{1}^{50} ; q_{1}^{50}\right)_{\infty}}{f\left(q_{1}^{5}, q_{1}^{45}\right)} \\
& -\left(\alpha^{\prime 2}+\alpha^{\prime 6}\right) q^{2} \frac{f\left(-q_{1}^{15}, q_{1}^{10}\right)\left(-q_{1}^{25} ;-q_{1}^{25}\right)_{\infty}\left(q_{1}^{50} ; q_{1}^{50}\right)_{\infty}}{f\left(q_{1}^{15}, q_{1}^{35}\right)}-2 \alpha^{\prime 4} q^{6} f\left(-q_{1}^{25},-q_{1}^{75}\right)^{2} .
\end{aligned}
$$

We also need the following results, which can be obtained by an easy calculation:

$$
\left\{\begin{array}{l}
1-2 \alpha^{\prime 4}+\alpha^{\prime 8}=i \sqrt{\frac{5+\sqrt{5}}{2}}\left(-\alpha^{\prime 2}+\alpha^{6}\right), \\
-1+\alpha^{\prime 2}+\alpha^{6}-\alpha^{\prime 8}=i \sqrt{\frac{5+\sqrt{5}}{2}}\left(1-\alpha^{\prime 8}\right) \text { or }-i \sqrt{\frac{5-\sqrt{5}}{2}}\left(-\alpha^{\prime 2}+\alpha^{6}\right), \\
\alpha^{\prime 2}-2 \alpha^{4}+\alpha^{\prime 6}=-i \sqrt{\frac{5-\sqrt{5}}{2}}\left(1-\alpha^{\prime 8}\right) .
\end{array}\right.
$$

Transforming $L_{1}$ and $L_{2}$ into the sums of $\psi(q)$ and theta functions, and $\phi(q)$ and theta functions with (1.7) and (1.8), using (4.8) and (4.10) to simplify the terms related to $\phi(q)$ and $\psi(q)$, replacing $q$ by $q_{1}$ in Theorem 2.19 , Theorem 2.20 , and Theorem 2.21, and applying Theorem 2.19, Theorem 2.20, and Theorem 2.21, we 
find that (4.9) equals

$$
\begin{aligned}
\sum_{m} & (-1)^{m} q_{1}^{m^{2}+m} \alpha^{2 m}\left(i \sqrt{\frac{5+\sqrt{5}}{2}} q_{1}^{-1} \psi\left(-q^{5}\right)-i \sqrt{\frac{5-\sqrt{5}}{2}} q_{1} \phi\left(-q^{5}\right)\right) \\
+ & \left(1+\alpha^{\prime 8}\right)\left(-q_{1} a_{1}\left(-q_{1}^{5}\right) f\left(-q_{1}^{10},-q_{1}^{40}\right)+2 q_{1}^{6} A\left(q_{1}^{30},-q_{1}^{5},-q_{1}^{25}\right)\right. \\
& -2 q_{1}^{12} A\left(q_{1}^{-20}, q_{1}^{10},-q_{1}^{25}\right)+q_{1}^{-1} a_{1}\left(-q_{1}^{5}\right) f\left(-q_{1}^{20},-q_{1}^{30}\right)+q_{1}^{3} a_{2}\left(-q_{1}^{5}\right) f\left(-q_{1}^{10},-q_{1}^{40}\right) \\
& \left.-2 q_{1}^{8} A\left(q_{1}^{20}, q_{1}^{10},-q_{1}^{25}\right)+\frac{f\left(-q_{1}^{5}, q_{1}^{20}\right)\left(-q_{1}^{25} ;-q_{1}^{25}\right)_{\infty}\left(q_{1}^{50} ; q_{1}^{50}\right)_{\infty}}{f\left(q_{1}^{5}, q_{1}^{55}\right)}\right) \\
+ & \left(\alpha^{\prime 2}+\alpha^{\prime 6}\right)\left(2 q_{1}^{10} A\left(q_{1}^{-10},-q_{1}^{5},-q_{1}^{25}\right)-q_{1}^{3} a_{2}\left(-q_{1}^{5}\right) f\left(-q_{1}^{10},-q_{1}^{40}\right)\right. \\
& -q_{1}^{-1} a_{1}\left(-q_{1}^{5}\right) f\left(-q_{1}^{20},-q_{1}^{30}\right)+2 q_{1}^{4} A\left(q_{1}^{10},-q_{1}^{5},-q_{1}^{25}\right)-q_{1} a_{2}\left(-q_{1}^{5}\right) f\left(-q_{1}^{20},-q_{1}^{30}\right) \\
& \left.+2 q_{1}^{6} A\left(q_{1}^{10}, q_{1}^{10},-q_{1}^{25}\right)-q^{2} \frac{f\left(-q_{1}^{15}, q_{1}^{10}\right)\left(-q_{1}^{25} ;-q_{1}^{25}\right)_{\infty}\left(q_{1}^{50} ; q_{1}^{50}\right)_{\infty}}{f\left(q_{1}^{15}, q_{1}^{35}\right)}\right) \\
+ & 2 \alpha^{\prime 4}\left(q_{1} a_{1}\left(-q^{5}\right) f\left(-q_{1}^{10},-q_{1}^{40}\right)+q_{1} a_{2}\left(-q_{1}^{5}\right) f\left(-q_{1}^{20},-q_{1}^{30}\right)-2 q_{1}^{6} f\left(-q_{1}^{25},-q_{1}^{75}\right)^{2}\right. \\
& \left.-q^{6} f\left(-q_{1}^{25},-q_{1}^{75}\right)^{2}\right) \\
= & \sum_{m}(-1)^{m} q_{1}^{m^{2}+m} \alpha^{\prime 2 m}\left(i \sqrt{\frac{5+\sqrt{5}}{2}} q_{1}^{-1} \psi\left(-q_{1}^{5}\right)-i \sqrt{\frac{5-\sqrt{5}}{2}} q_{1} \phi\left(-q_{1}^{5}\right)\right) \\
& +\left(1+\alpha^{\prime 8}\right) \frac{f\left(-q_{1},-q_{1}^{9}\right)^{2} f\left(-q_{1}^{8},-q_{1}^{12}\right)\left(q_{1}^{10} ; q_{1}^{10}\right)_{\infty}\left(q_{1}^{20} ; q_{1}^{20}\right)_{\infty}}{f\left(-q_{1}^{3},-q_{1}^{7}\right) f\left(-q_{1}^{2},-q_{1}^{18}\right) f\left(-q_{1}^{4},-q_{1}^{16}\right)} \\
& -\left(\alpha^{\prime 2}+\alpha^{\prime 6}\right) q_{1} \frac{f\left(-q_{1}^{3},-q_{1}^{7}\right)^{2} f\left(-q_{1}^{4},-q_{1}^{16}\right)\left(q_{1}^{10} ; q_{1}^{10}\right)_{\infty}\left(q_{1}^{20} ; q_{1}^{20}\right)_{\infty}}{f\left(-q_{1},-q_{1}^{9}\right) f\left(-q_{1}^{6},-q_{1}^{14}\right) f\left(-q_{1}^{8},-q_{1}^{12}\right)} \\
& +2 \alpha^{\prime 4} q_{1} f\left(-q_{1}^{5},-q_{1}^{15}\right)^{2} .
\end{aligned}
$$

By (4.7), (4.9), and (4.11), we find that

$$
\begin{aligned}
& -\frac{\sqrt{5-\sqrt{5}}}{5 n \sqrt{2}}\left(e^{\frac{\pi n}{5}} \frac{F\left(\frac{1}{5}, \frac{i}{5 n}\right)}{\theta_{11}(i n, 5 i n)}-e^{\frac{16 \pi n}{5}} \frac{F\left(\frac{4}{5}, \frac{i}{5 n}\right)}{\theta_{11}(4 i n, 5 i n)}\right) \\
& =-\frac{1}{\sqrt{n}} q_{1}^{-1} \psi\left(-q^{5}\right)+\frac{\sqrt{5}-1}{2 \sqrt{n}} q_{1} \phi\left(-q_{1}^{5}\right)+i \frac{\sqrt{5-\sqrt{5}}}{\sqrt{10 n}} \frac{1}{\sum_{m}(-1)^{m} q_{1}^{m^{2}+m} \alpha^{2 m}} \\
& \quad \times\left(\left(1+\alpha^{\prime 8}\right) \frac{f\left(-q_{1},-q_{1}^{9}\right)^{2} f\left(-q_{1}^{8},-q_{1}^{12}\right)\left(q_{1}^{10} ; q_{1}^{10}\right)_{\infty}\left(q_{1}^{20} ; q_{1}^{20}\right)_{\infty}}{f\left(-q_{1}^{3},-q_{1}^{7}\right) f\left(-q_{1}^{2},-q_{1}^{18}\right) f\left(-q_{1}^{4},-q_{1}^{16}\right)}\right. \\
& \quad-\left(\alpha^{\prime 2}+\alpha^{\prime 6}\right) q_{1} \frac{f\left(-q_{1}^{3},-q_{1}^{7}\right)^{2} f\left(-q_{1}^{4},-q_{1}^{16}\right)\left(q_{1}^{10} ; q_{1}^{10}\right)_{\infty}\left(q_{1}^{20} ; q_{1}^{20}\right)_{\infty}}{f\left(-q_{1},-q_{1}^{9}\right) f\left(-q_{1}^{6},-q_{1}^{14}\right) f\left(-q_{1}^{8},-q_{1}^{12}\right)} \\
& \left.\quad+2 \alpha^{\prime 4} q_{1} f\left(-q_{1}^{5},-q_{1}^{15}\right)^{2}\right) .
\end{aligned}
$$


By (1.3), (1.16), and (3.6), we derive the next two identities:

$$
\left\{\begin{array}{l}
f\left(-q^{3},-q^{7}\right)=-\frac{i}{\sqrt{5 n}} e^{\frac{n \pi}{5}-\frac{\pi}{20 n}+\frac{3 \pi i}{10}} \sum_{m}(-1)^{m} q_{1}^{m^{2}+m} e^{2 m \frac{3 \pi i}{10}} \\
f\left(q^{2}, q^{8}\right)=\frac{1}{\sqrt{5 n}} e^{\frac{9 n \pi}{20}} \sum_{m}(-1)^{m} q_{1}^{m^{2}} e^{2 m \frac{\pi i}{5}}
\end{array}\right.
$$

Using (3.22) and (4.13), we find that

$$
\begin{aligned}
& \frac{f\left(-q^{3},-q^{7}\right) f\left(q, q^{9}\right) f\left(q^{2}, q^{8}\right)}{f\left(q^{3}, q^{7}\right)}=-\frac{1}{5 n} e^{-\frac{\pi}{20 n}+\frac{25 n \pi}{20}} \\
& \times \frac{\alpha^{4} \sum_{m}(-1)^{m} q_{1}^{m^{2}+m} e^{2 m \frac{3 \pi i}{10}} \sum_{m}(-1)^{m} q_{1}^{m^{2}} e^{2 m \frac{\pi i}{10}} \sum_{m}(-1)^{m} q_{1}^{m^{2}} e^{2 m \frac{\pi i}{5}}}{\sum_{m}(-1)^{m} q_{1}^{m^{2}} e^{2 m \frac{3 \pi i}{10}}} .
\end{aligned}
$$

Recall that $\alpha^{\prime}=e^{\pi i / 5}$ and $\alpha^{\prime 5}=-1$. By (3.20), (3.21), Theorem 2.22 with $q$ replaced by $q_{1}$, and Theorem 2.6 with $q$ replaced by $q_{1}$, the numerator of the right side of (4.14) equals

$$
\begin{aligned}
& (4.15) \\
\alpha^{\prime 4} & \left(\left(1+\alpha^{2}\right) f\left(q_{1}^{20}, q_{1}^{30}\right)-\left(\alpha^{\prime 3}-\alpha^{4}\right) q_{1}^{2} f\left(q_{1}^{10}, q_{1}^{40}\right)-2 \alpha^{\prime} q_{1}^{6} f\left(q_{1}^{50}, q_{1}^{150}\right)\right) \\
& \times\left(f\left(q_{1}^{25}, q_{1}^{25}\right)-\left(\alpha^{\prime}-\alpha^{\prime 4}\right) q_{1} f\left(q_{1}^{15}, q_{1}^{35}\right)+\left(\alpha^{\prime 2}-\alpha^{33}\right) q_{1}^{4} f\left(q_{1}^{5}, q_{1}^{45}\right)\right) \\
& \times\left(f\left(-q_{1}^{25},-q_{1}^{25}\right)-\left(\alpha^{\prime 2}-\alpha^{\prime 3}\right) q_{1} f\left(-q_{1}^{15},-q_{1}^{35}\right)-\left(\alpha^{\prime}-\alpha^{4}\right) q_{1}^{4} f\left(-q_{1}^{5},-q_{1}^{45}\right)\right) \\
= & \alpha^{\prime 4}\left(\left(1+\alpha^{\prime 2}\right) f\left(q_{1}^{20}, q_{1}^{30}\right)-\left(\alpha^{\prime 3}-\alpha^{\prime 4}\right) q_{1}^{2} f\left(q_{1}^{10}, q_{1}^{40}\right)-2 \alpha^{\prime} q_{1}^{6} f\left(q_{1}^{50}, q_{1}^{150}\right)\right) \\
\times & \left(\frac{\left.f\left(-q_{1},-q_{1}^{9}\right)\left(q_{1}^{4} ; q_{1}^{4}\right)_{\infty}\left(q_{1}^{10} ; q_{1}^{10}\right)_{\infty}^{2}-2\left(\alpha^{\prime 2}-\alpha^{\prime 3}\right) q_{1} f\left(-q_{1}^{4},-q_{1}^{16}\right) f\left(-q_{1}^{8},-q_{1}^{12}\right)\right)}{f\left(-q_{1}^{3},-q_{1}^{7}\right) f\left(-q_{1}^{2},-q_{1}^{18}\right)}\right. \\
= & \left(-\alpha^{\prime}+\alpha^{\prime 4}\right) f\left(q_{1}^{20}, q_{1}^{30}\right) \frac{f\left(-q_{1},-q_{1}^{9}\right)\left(q_{1}^{4} ; q_{1}^{4}\right)_{\infty}\left(q_{1}^{10} ; q_{1}^{10}\right)_{\infty}^{2}}{f\left(-q_{1}^{3},-q_{1}^{7}\right) f\left(-q_{1}^{2},-q_{1}^{18}\right)} \\
& +2 q_{1} f\left(q_{1}^{20}, q_{1}^{30}\right) f\left(-q_{1}^{4},-q_{1}^{16}\right) f\left(-q_{1}^{8},-q_{1}^{12}\right) \\
& +\left(\alpha^{\prime 2}-\alpha^{\prime 3}\right) q_{1}^{2} f\left(q_{1}^{10}, q_{1}^{40}\right) \frac{f\left(-q_{1},-q_{1}^{9}\right)\left(q_{1}^{4} ; q_{1}^{4}\right)_{\infty}\left(q_{1}^{10} ; q_{1}^{10}\right)_{\infty}^{2}}{f\left(-q_{1}^{3},-q_{1}^{7}\right) f\left(-q_{1}^{2},-q_{1}^{18}\right)} \\
& -2\left(2-\alpha^{\prime}+\alpha^{\prime 4}\right) q_{1}^{3} f\left(q_{1}^{10}, q_{1}^{40}\right) f\left(-q_{1}^{4},-q_{1}^{16}\right) f\left(-q_{1}^{8},-q_{1}^{12}\right) \\
& +2 q_{1}^{6} f\left(q_{1}^{50}, q_{1}^{150}\right) \frac{f\left(-q_{1},-q_{1}^{9}\right)\left(q_{1}^{4} ; q_{1}^{4}\right){ }_{\infty}\left(q_{1}^{10} ; q_{1}^{10}\right)_{\infty}^{2}}{f\left(-q_{1}^{3},-q_{1}^{7}\right) f\left(-q_{1}^{2},-q_{1}^{18}\right)} \\
& -4\left(\alpha^{\prime 2}-\alpha^{\prime 3}\right) q_{1}^{7} f\left(q_{1}^{50}, q_{1}^{150}\right) f\left(-q_{1}^{4},-q_{1}^{16}\right) f\left(-q_{1}^{8},-q_{1}^{12}\right) .
\end{aligned}
$$

Here, we easily calculate that $-\alpha^{\prime}+\alpha^{4}=\frac{-1-\sqrt{5}}{2}, \alpha^{\prime 2}-\alpha^{\prime 3}=\frac{-1+\sqrt{5}}{2}$, and $2-$ $\alpha^{\prime}+\alpha^{\prime 4}=\frac{3-\sqrt{5}}{2}$. Let $L S_{1}^{\prime}=-\frac{1}{2} \times$ the left side of $(2.31)$ with $q$ replaced by $q_{1}$, $L S_{2}^{\prime}=-\frac{\sqrt{5}}{2} \times$ the left side of $(2.32)$ with $q$ replaced by $q_{1}, R S_{1}^{\prime}=-\frac{1}{2} \times$ the right side of (2.31) with $q$ replaced by $q_{1}$, and $R S_{2}^{\prime}=-\frac{\sqrt{5}}{2} \times$ the right side of (2.32) with $q$ replaced by $q_{1}$. Then, we easily verify that the right side of (4.16) equals the sum of $L S_{1}^{\prime}$ and $L S_{2}^{\prime}$. Therefore, we can say that the right side of (4.15) equals the sum 
of $R S_{1}^{\prime}$ and $R S_{2}^{\prime}$. Using (3.21), we find that the right side of (4.15) equals

$$
\begin{aligned}
& -\left(\left(\alpha^{\prime}-\alpha^{4}\right) f\left(q_{1}^{25}, q_{1}^{25}\right) \frac{f\left(-q_{1},-q_{1}^{9}\right)^{2} f\left(-q_{1}^{8},-q_{1}^{12}\right)\left(q_{1}^{10} ; q_{1}^{10}\right)_{\infty}\left(q_{1}^{20} ; q_{1}^{20}\right)_{\infty}}{f\left(-q_{1}^{3},-q_{1}^{7}\right) f\left(-q_{1}^{2},-q_{1}^{18}\right) f\left(-q_{1}^{4},-q_{1}^{16}\right)}\right. \\
& -2 q_{1} f\left(q_{1}^{25}, q_{1}^{25}\right) f\left(-q_{1}^{5},-q_{1}^{15}\right)^{2} \\
& +\left(\alpha^{\prime 2}-\alpha^{\prime 3}\right) q_{1} f\left(q_{1}^{25}, q_{1}^{25}\right) \frac{f\left(-q_{1}^{3},-q_{1}^{7}\right)^{2} f\left(-q_{1}^{4},-q_{1}^{16}\right)\left(q_{1}^{10} ; q_{1}^{10}\right)_{\infty}\left(q_{1}^{20} ; q_{1}^{20}\right)_{\infty}}{f\left(-q_{1},-q_{1}^{9}\right) f\left(-q_{1}^{6},-q_{1}^{14}\right) f\left(-q_{1}^{8},-q_{1}^{12}\right)} \\
& +q_{1} f\left(q_{1}^{15}, q_{1}^{35}\right) \frac{f\left(-q_{1},-q_{1}^{9}\right)^{2} f\left(-q_{1}^{8},-q_{1}^{12}\right)\left(q_{1}^{10} ; q_{1}^{10}\right)_{\infty}\left(q_{1}^{20} ; q_{1}^{20}\right)_{\infty}}{f\left(-q_{1}^{3},-q_{1}^{7}\right) f\left(-q_{1}^{2},-q_{1}^{18}\right) f\left(-q_{1}^{4},-q_{1}^{16}\right)} \\
& -2\left(\alpha^{\prime 2}-\alpha^{\prime 3}\right) q_{1}^{2} f\left(q_{1}^{15}, q_{1}^{35}\right) f\left(-q_{1}^{5},-q_{1}^{15}\right)^{2} \\
& +\left(2-\alpha^{\prime}+\alpha^{\prime 4}\right) q_{1}^{2} f\left(q_{1}^{15}, q_{1}^{35}\right) \frac{f\left(-q_{1}^{3},-q_{1}^{7}\right)^{2} f\left(-q_{1}^{4},-q_{1}^{16}\right)\left(q_{1}^{10} ; q_{1}^{10}\right)_{\infty}\left(q_{1}^{20} ; q_{1}^{20}\right)_{\infty}}{f\left(-q_{1},-q_{1}^{9}\right) f\left(-q_{1}^{6},-q_{1}^{14}\right) f\left(-q_{1}^{8},-q_{1}^{12}\right)} \\
& -\left(2+\alpha^{\prime 2}-\alpha^{\prime 3}\right) q_{1}^{4} f\left(q_{1}^{5}, q_{1}^{45}\right) \frac{f\left(-q_{1},-q_{1}^{9}\right)^{2} f\left(-q_{1}^{8},-q_{1}^{12}\right)\left(q_{1}^{10} ; q_{1}^{10}\right)_{\infty}\left(q_{1}^{20} ; q_{1}^{20}\right)_{\infty}}{f\left(-q_{1}^{3},-q_{1}^{7}\right) f\left(-q_{1}^{2},-q_{1}^{18}\right) f\left(-q_{1}^{4},-q_{1}^{16}\right)} \\
& +2\left(\alpha^{\prime}-\alpha^{4}\right) q_{1}^{5} f\left(q_{1}^{5}, q_{1}^{45}\right) f\left(-q_{1}^{5},-q_{1}^{15}\right)^{2} \\
& \left.-q_{1}^{5} f\left(q_{1}^{5}, q_{1}^{45}\right) \frac{f\left(-q_{1}^{3},-q_{1}^{7}\right)^{2} f\left(-q_{1}^{4},-q_{1}^{16}\right)\left(q_{1}^{10} ; q_{1}^{10}\right)_{\infty}\left(q_{1}^{20} ; q_{1}^{20}\right)_{\infty}}{f\left(-q_{1},-q_{1}^{9}\right) f\left(-q_{1}^{6},-q_{1}^{14}\right) f\left(-q_{1}^{8},-q_{1}^{12}\right)}\right) \\
& =-\alpha^{\prime}\left(f\left(q_{1}^{25}, q_{1}^{25}\right)+\left(\alpha^{\prime 2}-\alpha^{\prime 3}\right) q_{1} f\left(q_{1}^{15}, q_{1}^{35}\right)-\left(\alpha^{\prime}-\alpha^{\prime 4}\right) q_{1}^{4} f\left(q_{1}^{5}, q_{1}^{45}\right)\right) \\
& \times\left(\left(1+\alpha^{\prime 8}\right) \frac{f\left(-q_{1},-q_{1}^{9}\right)^{2} f\left(-q_{1}^{8},-q_{1}^{12}\right)\left(q_{1}^{10} ; q_{1}^{10}\right)_{\infty}\left(q_{1}^{20} ; q_{1}^{20}\right)_{\infty}}{f\left(-q_{1}^{3},-q_{1}^{7}\right) f\left(-q_{1}^{2},-q_{1}^{18}\right) f\left(-q_{1}^{4},-q_{1}^{16}\right)}\right. \\
& -\left(\alpha^{\prime 2}+\alpha^{\prime 6}\right) q_{1} \frac{f\left(-q_{1}^{3},-q_{1}^{7}\right)^{2} f\left(-q_{1}^{4},-q_{1}^{16}\right)\left(q_{1}^{10} ; q_{1}^{10}\right)_{\infty}\left(q_{1}^{20} ; q_{1}^{20}\right)_{\infty}}{f\left(-q_{1},-q_{1}^{9}\right) f\left(-q_{1}^{6},-q_{1}^{14}\right) f\left(-q_{1}^{8},-q_{1}^{12}\right)} \\
& \left.+2 \alpha^{4} q_{1} f\left(-q_{1}^{5},-q_{1}^{15}\right)^{2}\right) \\
& =-\alpha^{\prime} \sum_{m} q_{1}^{m^{2}} e^{2 m \frac{3 \pi i}{10}}\left(\left(1+\alpha^{\prime 8}\right) \frac{f\left(-q_{1},-q_{1}^{9}\right)^{2} f\left(-q_{1}^{8},-q_{1}^{12}\right)\left(q_{1}^{10} ; q_{1}^{10}\right)_{\infty}\left(q_{1}^{20} ; q_{1}^{20}\right)_{\infty}}{f\left(-q_{1}^{3},-q_{1}^{7}\right) f\left(-q_{1}^{2},-q_{1}^{18}\right) f\left(-q_{1}^{4},-q_{1}^{16}\right)}\right. \\
& -\left(\alpha^{\prime 2}+\alpha^{\prime 6}\right) q_{1} \frac{f\left(-q_{1}^{3},-q_{1}^{7}\right)^{2} f\left(-q_{1}^{4},-q_{1}^{16}\right)\left(q_{1}^{10} ; q_{1}^{10}\right)_{\infty}\left(q_{1}^{20} ; q_{1}^{20}\right)_{\infty}}{f\left(-q_{1},-q_{1}^{9}\right) f\left(-q_{1}^{6},-q_{1}^{14}\right) f\left(-q_{1}^{8},-q_{1}^{12}\right)} \\
& \left.+2 \alpha^{\prime 4} q_{1} f\left(-q_{1}^{5},-q_{1}^{15}\right)^{2}\right)
\end{aligned}
$$

By (4.5), (1.3), and (1.16), we find that

$$
f\left(-q^{2},-q^{8}\right)=\frac{\sum_{m}(-1)^{m} q_{1}^{m^{2}+m} \alpha^{\prime 2 m}}{i \sqrt{5 n} \alpha^{\prime-1} e^{\frac{\pi}{20 n}} e^{-\frac{9 n \pi}{20}}} .
$$


By (4.16) and (4.17), we find that

$$
\begin{aligned}
& -\sqrt{\frac{5-\sqrt{5}}{2}} e^{\frac{\pi n}{5}} q \frac{f\left(-q^{3},-q^{7}\right) f\left(q, q^{9}\right) f\left(q^{2}, q^{8}\right)}{f\left(-q^{2},-q^{8}\right) f\left(q^{3}, q^{7}\right)} \\
& =\sqrt{\frac{5-\sqrt{5}}{2}} \frac{1}{5 n} e^{-\frac{\pi}{20 n}+\frac{9 n \pi}{20}} i \sqrt{5 n} \alpha^{\prime-1} e^{\frac{\pi}{20 n}} e^{-\frac{9 n \pi}{20}} \\
& \times \frac{\alpha^{\prime 4} \sum_{m}(-1)^{m} q_{1}^{m^{2}+m} e^{2 m \frac{3 \pi i}{10}} \sum_{m}(-1)^{m} q_{1}^{m^{2}} e^{2 m \frac{\pi i}{10}} \sum_{m}(-1)^{m} q_{1}^{m^{2}} e^{2 m \frac{\pi i}{5}}}{\sum_{m}(-1)^{m} q_{1}^{m^{2}+m} \alpha^{2 m} \sum_{m}(-1)^{m} q_{1}^{m^{2}} e^{2 m \frac{3 \pi i}{10}}} \\
& =-\sqrt{\frac{5-\sqrt{5}}{10 n}} \frac{i}{\sum_{m}(-1)^{m} q_{1}^{m^{2}+m} \alpha^{\prime 2 m}} \\
& \quad \times\left(\left(1+\alpha^{\prime 8}\right) \frac{f\left(-q_{1},-q_{1}^{9}\right)^{2} f\left(-q_{1}^{8},-q_{1}^{12}\right)\left(q_{1}^{10} ; q_{1}^{10}\right)_{\infty}\left(q_{1}^{20} ; q_{1}^{20}\right)_{\infty}}{f\left(-q_{1}^{3},-q_{1}^{7}\right) f\left(-q_{1}^{2},-q_{1}^{18}\right) f\left(-q_{1}^{4},-q_{1}^{16}\right)}\right. \\
& \quad-\left(\alpha^{\prime 2}+\alpha^{\prime 6}\right) q_{1} \frac{f\left(-q_{1}^{3},-q_{1}^{7}\right)^{2} f\left(-q_{1}^{4},-q_{1}^{16}\right)\left(q_{1}^{10} ; q_{1}^{10}\right)_{\infty}\left(q_{1}^{20} ; q_{1}^{20}\right)_{\infty}}{f\left(-q_{1},-q_{1}^{9}\right) f\left(-q_{1}^{6},-q_{1}^{14}\right) f\left(-q_{1}^{8},-q_{1}^{12}\right)} \\
& \left.+2 \alpha^{\prime 4} q_{1} f\left(-q_{1}^{5},-q_{1}^{15}\right)^{2}\right) \cdot
\end{aligned}
$$

In conclusion, using (4.3), (4.4), (4.12), and (4.18), we have completed the proof of Ramanujan's eighth identity.

\section{REFERENCES}

[AH] G. E. Andrews and D. Hickerson, Ramanujan's "Lost" Notebook VII:The sixth order mock theta functions, Adv. Math. 89 (1991), 60-105. MR 92i:11027

[B1] B. C. Berndt, Ramanujan's Notebooks Part III, Springer-Verlag, New York, 1991. MR 92j:01069

[B2] B. C. Berndt, Ramanujan's Notebooks Part IV, Springer-Verlag, New York, 1994. MR 95e: 11028

[BR] B. C. Berndt and R. A. Rankin, Ramanujan: Letters and Commentary, Amer. Math. Soc., Providence, 1995; London Math. Soc., London, 1995. MR 97c:01034

[C1] Y.-S. Choi, Tenth order mock theta functions in Ramanujan's Lost Notebook, Invent. Math. 136 (1999), 497-569. MR 2000f: 11016

[C2] Y.-S. Choi, Tenth order mock theta functions in Ramanujan's Lost Notebook (II), Adv. Math. 156 (2000), 180-285. CMP 2001:07

[C3] Y.-S. Choi, Two identities for tenth order mock theta functions in Ramanujan's Lost Notebook, submitted for publication.

[GR] G. Gasper and M. Rahman, Basic Hypergeometric Series, Cambridge University Press, Cambridge, 1990. MR 91d:33034

[H1] D. Hickerson, A proof of the mock theta conjectures, Invent. Math. 94 (1988), 639-660. MR 90f:11028a

[H2] D. Hickerson, On the seventh order mock theta functions, Invent. Math. 94 (1988), 661-677. MR 90f:11028b

[KM] M. I. Knopp, Modular Functions in Analytic Number Theory, reprint, Chelsea Publishing Co., New York, 1993. MR 42:198 (original ed.)

[ML] L. J. Mordell, The value of the definite integral, Quarterly Journal of Mathematics 48 (1920), 329-342.

[RA] S. Ramanujan, The Lost Notebook and Other Unpublished papers, Narosa Publishing House, New Delhi, 1988. MR 89j:01078

[RR] R. A. Rankin, Modular Forms and Functions, Cambridge University Press, Cambridge, 1977. MR 58:16518 
[RS] S. Robins, Generalized Dedekind $\eta$-products, Contemp. Math. 166 (1994), 119-128. MR 95k:11061

[WG] G. N. Watson, The final problem:An account of the mock theta functions, J. London Math. Soc. 11 (1936), 55-80.

Department of Mathematics, Korea University, 5-1, Anam-dong, Sungbuk-ku, Seoul, 136-701, KOREA

E-mail address: y-choi2@mail.korea.ac.kr 